\title{
Thermal bosonisation in the sine-Gordon and massive Thirring models
}

\author{
A. Gómez Nicola ${ }^{a, 1}$, D.A. Steer ${ }^{b, 2,3}$ \\ ${ }^{a}$ Departamento de Fisica Teórica, Universidad Complutense, 28040, Madrid, Spain \\ b D.A.M.T.P., Silver Street, Cambridge, CB3 9EW, UK
}

Received 2 November 1998; accepted 26 February 1999

\begin{abstract}
We study bosonisation in the massive Thirring and sine-Gordon models at finite temperature $T$ and non-zero fermion chemical potential $\mu$. For that purpose we use both canonical operator and path-integral approaches, paying particular attention to the issues of thermal normal ordering and renormalisation. At $T>0$ and $\mu=0$, the massive Thirring model bosonises to the sineGordon model with the same $T=0$ identification between coupling constants. We prove that not only the partition functions of the two models coincide, as was recently shown, but also that thermal averages of zero-charge operators can be identified. In particular, analysis of the pointsplit regularised fermion current then leads to the thermal equivalence between sine-Gordon kinks and Thirring fermions. At $\mu \neq 0, T>0$ and working in perturbation theory about the massless Thirring model, we show that the bosonised theory is the sine-Gordon model plus an additional topological term which accounts for the existence of net fermion charge excitations (the fermions or the kinks) in the thermal bath. This result generalises one recently obtained for the massless case, and it is the two-dimensional version of the low-energy QCD chiral Lagrangian at finite baryon density. (C) 1999 Elsevier Science B.V. All rights reserved.
\end{abstract}

\footnotetext{
'E-mail: gomez@eucmax.sim.ucm.es

${ }^{2}$ E-mail: d.a.steer@damtp.cam.ac.uk

${ }^{3}$ Corresponding author.
} 


\section{Introduction}

The sine-Gordon (SG) and massive Thirring (MT) models are just two of many different models which have been widely studied in two space-time dimensions (2D). They provide one of the simplest examples of bosonisation, in which a fermion theory (MT) is equivalent to a boson one (SG) with certain identifications between their coupling constants. Furthermore the weak limit of one theory is the strong limit of the other and conversely so that these models also give a simple example of duality, and the particle spectrum can be mapped from one model onto the other. This equivalence was first analysed in [1] using canonical operator methods and, later on, using path integral in $[2,3]$. In the MT-SG system, bosonisation takes place for the full spectrum. This should be contrasted, for instance, to QCD where it happens only at low energies for which strongly coupled quarks and gluons are confined into hadrons. There the effective boson theory is the chiral Lagrangian for the lightest mesons which are the Nambu-Goldstone bosons of the chiral symmetry breaking pattern, and to lowest order it is the non-linear sigma model [4].

It is important to note that the particle spectrum of the bosonised theory may still contain fermion-like excitations. In the SG model, such excitations are soliton-like, corresponding classically to non-dissipative solutions of the equations of motion. One can assign a conserved charge to those configurations, corresponding to the fermion charge [5] which is a super-selection rule, so that the theory can be classified into different charge sectors. For instance, a possible one-fermion solution is the static kink [6]. The particle spectrum of the SG theory is then constructed from the mesons (including kink-anti-kink 'breather solutions') and the kinks, which are in one to one correspondence with the Thirring model fermion-antifermion bound states and fermions, respectively. The situation is entirely analogous to low-energy QCD where the mesons are the pions, kaons and so on and the solitons are the skyrmions [7], corresponding physically to the QCD baryons [8]. An example in which the bosonised theory does not contain fermion modes is the Schwinger model (QED in 2D) which bosonises to a free massive scalar with mass proportional to the electric charge [9]. This scalar is a fermion-antifermion bound state in which the electric charge is confined.

The link with QCD discussed above is just one of the motivations to study these 2D theories as toy models. However, one should recall that some theories in $2 \mathrm{D}$ also describe experimentally observed phenomena. Indeed, the SG equation is behind a device which produces some of the highest energy microwave signals available, having technological applications, for example, in space physics [10,11]. Such Josephson junctions consist of two layers of super-conducting materials separated by a very thin dielectric barrier, and Josephson tunnelling of the Cooper pairs across this barrier results in the relative phase angle of the two superconductors satisfying a $S G$ equation [10]. As the two superconductors are taken through the phase transition, kinks are formed in the junction and these are observed experimentally [12]. The kinks are topological defects, the 2D analogues of cosmic strings or vortices, line-like defects formed in $4 \mathrm{D}$ when a system goes through a symmetry breaking phase transition (say of some group $G$ to a subgroup 
$H$ ) for which the first homotopy group $\pi_{1}(G / H) \neq 0[13,14]$. In the early universe they may provide an explanation for the formation of structures such as galaxies [14]. An important problem in this context is to estimate the number density of such strings formed at the phase transition and also their length distribution. Recently progress has been made by using the analogy between experimentally observable systems such as ${ }^{3} \mathrm{He}$ and ${ }^{4} \mathrm{He}$ and the early universe [15]. It would seem, however, that Josephson junctions provide an even more simple experimentally accessible system with which one could try to test ideas of defect formation. Furthermore the analysis should be simplified in this case by exploiting the duality between the SG and the MT models [16]. For that purpose, one needs to know first what is the exact relation between these two models at $T>0$ and $\mu \neq 0$, and how kink densities and fermion densities are related (see Section 5 below).

In this paper we therefore study bosonisation in the SG and MT models at finite temperature $T$ and non-zero chemical potential $\mu$. Recall that regarding bosonisation, temperature and chemical potentials play a completely different rôle. Temperature affects the boundary conditions of the propagator and hence bosonisation implies a change from Fermi-Dirac to Bose-Einstein statistics. However, a non-zero chemical potential allows the system to have net charged states. As we have commented before, this is especially relevant when the bosonised theory admits fermion states as in the SG model or in chiral Lagrangians. In fact, we expect the chemical potential to reflect that property. Besides, $\mu \neq 0$ spoils the hermiticity of the Dirac operator and breaks charge conjugation $(C)$ invariance. Indeed breaking the combination of $C$ and Lorentz covariance (the thermal bath selects a preferred frame) can give rise to topological $\mu$-dependent new terms in the bosonised action [17-19].

We now introduce the models. In Minkowski space with metric $(+,-)$, the Lagrangian densities for the SG and massive Thirring models are respectively

$$
\begin{aligned}
\mathcal{L}_{\mathrm{SG}}[\phi] & =\frac{1}{2} \partial_{\mu} \phi \partial^{\mu} \phi+\frac{\alpha_{0}}{\lambda^{2}} \cos \lambda \phi-\gamma_{0}, \\
\mathcal{L}_{\mathrm{MT}}[\bar{\psi}, \psi] & =i \bar{\psi}\left(\not \partial-m_{0}\right) \psi+\frac{1}{2} g^{2} j_{\mu}(x) j^{\mu}(x),
\end{aligned}
$$

where $j_{\mu}(x)=\bar{\psi}(x) \gamma_{\mu} \psi(x), \alpha_{0}, m_{0}$ and $\gamma_{0}$ are bare parameters and $\phi$ and $\psi$ are bosonic and fermionic fields respectively. The Minkowski gamma matrices are

$$
\gamma_{0}=\left(\begin{array}{ll}
0 & 1 \\
1 & 0
\end{array}\right), \quad \gamma_{1}=\left(\begin{array}{cc}
0 & 1 \\
-1 & 0
\end{array}\right), \quad \gamma_{5}=\gamma_{0} \gamma_{1}=\left(\begin{array}{cc}
-1 & 0 \\
0 & 1
\end{array}\right)
$$

so that

$$
\left\{\gamma^{\mu}, \gamma^{\nu}\right\}=2 g^{\mu \nu}, \quad \gamma_{\mu} \gamma_{5}=-\epsilon_{\mu \nu} \gamma^{\nu}
$$

with $\epsilon_{01}=1$.

As we have commented before, the $S G$ equations of motion admit non-dissipative solutions for which the classical field $\phi_{c l}(t, \pm \infty)=2 \pi n_{ \pm} / \lambda$, with $n_{ \pm} \in \mathbb{Z}$ (with properly normalised $\gamma_{0}$ ). In fact, those classical static solutions with $n_{+} \neq n_{-}$are the kinks we discussed above. It is also worth noticing that the SG Lagrangian in (1.1) 
corresponds to a non-linear sigma model in 2D for a single Nambu-Goldstone-like field $\phi$. Although there is no spontaneous symmetry breaking in $2 \mathrm{D}[20]$, the potential term in (1.1) breaks explicitly the symmetry $\phi \rightarrow \phi+a$ with $a \in \mathbb{R}$ (which we will call the chiral symmetry for reasons to become clear below) still preserving the symmetry $\phi \rightarrow \phi+2 \pi n / \lambda$ with $n \in \mathbb{Z}$. These two symmetries are, respectively, the counterparts of the chiral and isospin symmetries for $\mathrm{QCD}, \alpha_{0}$ and $\lambda$ playing the rôle of the pion mass squared and the inverse of the pion decay constant respectively. On the other hand, the chiral symmetry transformations in terms of the Thirring fermion are $\psi \rightarrow \exp \left(i a \lambda \gamma_{5}\right) \psi$. The massless Thirring model is chirally invariant, the fermion mass term breaking that symmetry in the same way as the $\alpha$ term does in the SG Lagrangian.

We now recall the results at $T=\mu=0$. In [1], Coleman, using a canonical field operator approach, considered chiral invariant combinations of zero-charge operators ${ }^{4}$ and showed that the SG and the massive Thirring models are equivalent in the following sense:

$$
\begin{aligned}
m \bar{\psi}(x) \psi(x) & =\frac{\alpha}{\lambda^{2}} \cos \lambda \phi(x), \\
j_{\mu}(x) & =\frac{\lambda}{2 \pi} \epsilon_{\mu \nu} \partial_{\nu} \phi(x),
\end{aligned}
$$

provided the constants of the two models are related through

$$
\begin{aligned}
& \frac{\lambda^{2}}{4 \pi}=\frac{1}{1+g^{2} / \pi}, \\
& \frac{\alpha}{\lambda^{2}}=\rho m,
\end{aligned}
$$

where $\rho$ is the renormalisation scale and all the coupling constants and fields in (1.3)(1.4) are renormalised (see Sections 2.2 and 3.2). In fact the renormalisation convention chosen here is not exactly the same as that of [1] (see below) and it also differs from [21] by a $\pi$ factor which may be absorbed in $\rho \rightarrow \rho / \pi$ in the above equation. All the physical results must be independent of the scale. Throughout this paper, we will refer to (1.3) and (1.4) as the mass and current equivalences respectively. It is very important to observe that whilst it may be inferred from [1] that the above equivalences are strong, this is not the case-they are weak identities, only holding between the vacua of the two theories. We also recall that (1.4) allows one to establish that the net fermion charge (fermions minus anti-fermions) in the massive Thirring model corresponds to the net kink number (kinks minus anti-kinks) in the SG model ${ }^{5}[1,5]$. Eqs. (1.5), (1.6) were also obtained using path-integral techniques in [2,3]. In both [1] and [2,3], the approach followed was to expand formally around the $\alpha_{0}=0$ in the SG model and the $m_{0}=0$ in the Thirring model-we will do the same here. An alternative approach

\footnotetext{
${ }^{4}$ These operators have the same number of fermions and anti-fermions in the MT, and correspond to operators which are invariant under $\phi \rightarrow \phi+2 \pi n / \lambda$, with $n \in \mathbb{Z}$, in the SG model.

${ }^{5}$ Note that here we are calling quantum configurations with any non-zero net charge and space-time dependence kinks, although strictly speaking the kinks are just time-independent classical configurations with net charge equal to one.
} 
would be, for instance, to work within a semiclassical approximation $[1,22]$, which we will not attempt in this work.

A question which may be asked is whether the equivalences (1.3)-(1.4) also hold in a heat bath where the vacuum expectation values are replaced by thermal expectation values. For bosons,

$$
\left\langle\langle\bullet\rangle=\frac{1}{Z(T)} \operatorname{Tr}\left(\bullet e^{-\beta \hat{H}}\right)=\frac{N_{\beta}}{Z(T)} \int_{\text {periodic }} d \phi \bullet \exp (-S[\phi]),\right.
$$

where $N_{\beta}$ is an infinite $T$-dependent constant arising in the path-integral description [23], $\beta=1 / T$, the path-integral boson fields are periodic in Euclidean time $\phi(\bar{t}+\beta)=$ $\phi(\bar{t})$ with $\bar{t}=$ it and

$$
Z(T)=\operatorname{Tr}\left(e^{-\beta \hat{H}}\right)=N_{\beta} \int_{\text {periodic }} d \phi \exp (-S[\phi])
$$

is the partition function. Thermal averages and partition functions for fermions are defined analogously, but integrating over anti-periodic field configurations instead. It is important to remember that for $\mu=0$, one works in the canonical ensemble, fixing the net fermion charge to zero. Consistently, in that case, the above trace is taken in the SG model over all possible states with zero net kink number or, equivalently, over field configurations that vanish at spatial infinity (configurations such as a kink-antikink pair are permitted). On the other hand, for $\mu \neq 0$, we are working in the grandcanonical ensemble and the net fermion number is not fixed. Hence the net averaged fermion density is non-zero and we are summing over all possible states in all the superselection sectors of the theory, i.e. over all possible net kink numbers in the SG model. In other words, the complete set of states over which the trace is taken can be chosen to be eigenstates of the Hamiltonian for $\mu=0$ and eigenstates of both the Hamiltonian and the number operator for $\mu \neq 0$. In Ref. [24] path-integral methods were used to show that the SG and MT model partition functions are equivalent at $T>0$ but $\mu=0$. On the other hand, it was shown in [19] that for $m_{0}=0$ and $\mu \neq 0$, the Thirring model partition function is not the free boson one $\left(\alpha_{0}=0\right)$, but it acquires an extra $\mu$-dependent term accounting for the non-zero fermion net number in the thermal bath.

In this work we analyse several aspects of the thermal bosonisation for this system which we believe have not been studied before. In the first part of the paper (Section 2) we extend the work of Coleman [1] to $T>0$ and $\mu=0$, working with a canonical operator approach. Particular attention is paid to the question of renormalisation and the definition of normal ordering in a heat bath. In the process, the results of [24] are reproduced though the approach is entirely different. The rest of the paper uses path-integral methods, which are introduced in Section 3. There we again prove that the partition functions of the two models are the same, but we pay special attention to renormalisation and to the connection with our results in the canonical formalism. The main objective of Section 4 is to prove that thermal averages of correlators of the zero-charge operators $(1.3),(1.4)$ evaluated at different space-time points coincide. 
Such correlators cannot be inferred in general from the partition function which contains information about global thermodynamic observables such as the pressure or the condensates, but not about correlators, which physically yield for example thermal correlation lengths. In our case, the thermal version of (1.4) will constitute the thermal Thirring fermion-SG kink equivalence. This equivalence will appear again in Section 5, where we analyse the case $T>0$ and $\mu \neq 0$ for the partition function, thus extending [19] to the massive case and [24] to $\mu \neq 0$. The massless and $\mu=0$ case was analysed in [25]. A summary of the results presented in this paper may be found in [26].

\section{Bosonisation in the canonical operator approach at $T>0$ and $\mu=0$}

Here we work in Minkowski space with a canonical operator approach and extend the results of [1] to $T>0$ through the use of thermal normal ordering introduced in [27] and defined below. Calculations are carried out in perturbation theory, expanding about $\alpha_{0}=0$ in the SG model and about $m_{0}=0$ in the Thirring model. We first summarise useful results for $2 \mathrm{D}$ free partition functions and propagators at finite temperature and zero chemical potential.

\subsection{Results for free fields in $2 D$ at $T>0$ and $\mu=0$}

\subsubsection{Notation and free partition functions}

For simplicity we take the spatial dimension to be finite $0 \leqslant x \leqslant L$ with corresponding discrete momenta $k:=k_{n}=2 \pi n / L$. The infinite length limit is reached by substituting $\sum_{k} \longrightarrow L \int_{-\infty}^{+\infty} d k / 2 \pi$. As opposed to the fields in the path integral (Sections 3-5), field operators are entirely independent of temperature $T=\beta^{-1}$ and hence do not satisfy any periodicity conditions in imaginary time. ${ }^{6}$ As usual, all the thermodynamic observables are obtained from the partition function and its derivatives by dividing by $\beta L$.

Consider a free real scalar field $\hat{\phi}$ of mass $\tilde{\mu}$ and a free massless fermion field $\hat{\psi}$ with corresponding Hamiltonians

$$
\hat{H}_{0}^{\bar{\mu}}=\int_{0}^{L} d x\left[\frac{\hat{\pi}^{2}}{2}+\frac{1}{2}\left(\frac{\partial \hat{\phi}}{\partial x}\right)^{2}+\frac{\tilde{\mu}^{2} \hat{\phi}^{2}}{2}\right], \quad \hat{H}_{0}^{F}=-i \int_{0}^{L} d x\left[\overline{\hat{\psi}} \gamma^{1} \partial_{1} \hat{\psi}\right],
$$

where $\hat{\pi}=\partial_{0} \hat{\phi}, \overline{\hat{\psi}}=\hat{\psi}^{\dagger} \gamma_{0}$ and the subscript 0 denotes the free case. As usual, the free field operators (or more generally operators in the interaction picture) are written in terms of annihilation and creation operators as

$$
\hat{\phi}(t, x)=\sum_{k}\left(2 \omega_{k, \tilde{\mu}} L\right)^{-1 / 2}\left[\hat{a}_{k, \tilde{\mu}} e^{-i k \cdot x}+\hat{a}_{k, \tilde{\mu}}^{\dagger} e^{i k \cdot x}\right],
$$

\footnotetext{
${ }^{6}$ Temperature dependence, and thus questions of periodicity, only appear when considering thermal expectation values of operators.
} 


$$
\hat{\psi}(t, x)=\sum_{k} L^{-1 / 2}\left[\hat{c}_{k} e^{-i k \cdot x}+\hat{d}_{k}^{\dagger} e^{i k \cdot x}\right] u(k)
$$

where $\omega_{k, \tilde{\mu}}^{2}=k^{2}+\tilde{\mu}^{2}$ and the two-vector $u(k)$ is

$$
u(k)=\left(\begin{array}{c}
\theta(-k) \\
\theta(k)
\end{array}\right)
$$

Also $k \cdot x$ is shorthand for $k_{0} t-k x$ with $k_{0}=\omega_{k, \tilde{\mu}}$, and

$$
\left[\hat{a}_{k, \tilde{\mu}}, \hat{a}_{k^{\prime}, \tilde{\mu}}^{\dagger}\right]=\left\{\hat{c}_{k}, \hat{c}_{k^{\prime}}^{\dagger}\right\}=\left\{\hat{d}_{k}, \hat{d}_{k^{\prime}}^{\dagger}\right\}=\delta_{k, k^{\prime}}
$$

with all other commutators and anti-commutators vanishing. Note that for the scalar field, our notation differs from the conventional one in that the annihilation and creation operators carry an extra label which is the mass $\tilde{\mu}$ appearing in the free Hamiltonian. In terms of annihilation and creation operators, the boson Hamiltonian is as usual,

$$
\hat{H}_{0}^{\bar{\mu}}=\sum_{k} \frac{\omega_{k, \tilde{\mu}}}{2}\left[\hat{a}_{k, \tilde{\mu}} \hat{a}_{k, \tilde{\mu}}^{\dagger}+\hat{a}_{k, \tilde{\mu}}^{\dagger} \hat{a}_{k, \tilde{\mu}}\right]=: \hat{H}_{0}^{\tilde{\mu}}: \bar{\mu}+\sum_{k} \frac{\omega_{k, \tilde{\mu}}}{2},
$$

where the standard normal ordering operation, : $\bullet: \bar{\mu}$, which places the annihilation operators $\hat{a}_{k, \tilde{\mu}}$ to the right of creation operators $\hat{a}_{k, \tilde{\mu}}^{\dagger}$, has been used to separate out the infinite vacuum energy. Note again the mass label on the normal ordering operation-it will be important below where objects such as : $\hat{H}_{0}^{\tilde{\mu}}: \rho$ with $\rho \neq \tilde{\mu}$ are considered. (By this we mean that the operators $\hat{\phi}$ must be expressed in terms of a mass $\rho$ in (2.2), and then the creation and annihilation operators $\hat{a}_{k, \rho}$ are ordered. This is equivalent to considering a mass shift in the Hamiltonian: $\hat{H}_{0}^{\tilde{\mu}}=\hat{H}_{0}^{\rho}+\left(\tilde{\mu}^{2}-\rho^{2}\right) \hat{\phi}^{2} / 2$.) From (2.3), the free boson partition function is, in the limit $L \rightarrow \infty$,

$$
\log Z_{0}^{\tilde{\mu}, B}(T)=\log \left[\operatorname{Tr}\left\{e^{-\beta \hat{H}_{0}^{\tilde{\mu}}}\right\}\right]=-L \int_{-\infty}^{+\infty} \frac{d k}{2 \pi}\left[\frac{\beta \omega_{k, \tilde{\mu}}}{2}+\log \left(1-e^{-\beta \omega_{k, \tilde{\mu}}}\right)\right] .
$$

The first term in the far r.h.s above is divergent and corresponds to the vacuum energy. In the canonical approach it is renormalised by normal ordering. In path-integral methods there is no operator normal ordering and infinite vacuum terms are not removed, although they are irrelevant for the thermodynamics (they are $T$-independent) and can be ignored [23]. For the massless fermion Hamiltonian one has

$$
\hat{H}_{0}^{F}=\sum_{k}|k|\left(\hat{c}_{k}^{\dagger} \hat{c}_{k}-\hat{d}_{k} \hat{d}_{k}^{\dagger}\right)=\sum_{k}|k|\left(\hat{c}_{k}^{\dagger} \hat{c}_{k}+\hat{d}_{k}^{\dagger} \hat{d}_{k}\right)-\sum_{k}|k|=: \hat{H}_{0}:-\sum_{k}|k|,
$$

whence

$$
\log Z_{0}^{F}=2 L \int_{-\infty}^{+\infty} \frac{d k}{2 \pi}\left[\frac{\beta|k|}{2}+\log \left(1+e^{-\beta|k|}\right)\right]
$$


Note that since

$$
\int_{-\infty}^{+\infty} \frac{d k}{2 \pi} \log \left(1-e^{-\beta|k|}\right)=-2 \int_{-\infty}^{+\infty} \frac{d k}{2 \pi} \log \left(1+e^{-\beta|k|}\right)=-\frac{\pi T}{6},
$$

it follows that, ignoring vacuum terms,

$$
Z_{0}^{F}(T)=Z_{0}^{0, B}(T)=\exp \left[\frac{\pi L T}{6}\right] .
$$

This result provides one of the simplest examples of bosonisation, and we will make use of it later on since it forms the basis of the equivalence of the SG and MT partition functions at $T>0$.

\subsubsection{Thermal normal ordering and thermal propagators}

Central to our discussion below will be the use of 'thermal normal ordering' [27]. The reason one is lead to consider a different normal ordering at $T>0$ comes from the fact that whilst at zero temperature, dealing with normal ordered products is easy as by construction their vacuum expectation value vanishes, for example,

$$
\left\langle\left\langle 0, \tilde{\mu}\left|: \hat{a}_{k, \tilde{\mu}} \hat{a}_{k, \tilde{\mu}}^{\dagger}: \tilde{\mu}\right| 0, \tilde{\mu}\right\rangle\right\rangle=0,
$$

this is no longer the case at $T>0$ where

$$
\left\langle\left\langle: \hat{a}_{k, \tilde{\mu}} \hat{a}_{k, \tilde{\mu}}^{\dagger}: \tilde{\mu}\right\rangle\right\rangle_{0}=\frac{\sum_{n=0}^{\infty}\left\langle\left\langle n, \tilde{\mu}\left|e^{-\beta: \hat{H}_{0}^{\tilde{\mu}}: \tilde{\mu}} \hat{a}_{k, \tilde{\mu}}^{\dagger} \hat{a}_{k, \tilde{\mu}}\right| n, \tilde{\mu}\right\rangle\right\rangle}{\sum_{n=0}^{\infty}\left\langle\left\langle n, \tilde{\mu}\left|e^{-\beta: \hat{H}_{0}^{\tilde{\mu}}: \tilde{\mu}}\right| n, \tilde{\mu}\right\rangle\right\rangle}=\frac{1}{e^{\beta \omega_{k, \tilde{\mu}}}-1}=N_{k, \tilde{\mu}} \neq 0 .
$$

Here $N_{k, \tilde{\mu}}$ is the Bose-Einstein distribution. As was discussed in [27,28], this problem can be avoided by introducing a more general and indeed more convenient definition of normal ordering than the one given above. It is obtained by splitting the field operators into two arbitrary parts $\hat{\Psi}=\hat{\Psi}^{+}+\hat{\Psi}^{-}(+/-$do not refer to positive/negative energy waves) with normal ordering $N^{\mathrm{ES}}$ defined to place the 'positive' parts to the right of the 'negative' parts so that, for example,

$$
N^{\mathrm{ES}}\left[\hat{\Psi}_{1} \hat{\Psi}_{2}\right]=\hat{\Psi}_{1}^{+} \hat{\Psi}_{2}^{+}+\hat{\Psi}_{1}^{-} \hat{\Psi}_{2}^{+}+\sigma \hat{\Psi}_{2}^{-} \hat{\Psi}_{1}^{+}+\hat{\Psi}_{1}^{-} \hat{\Psi}_{2}^{-},
$$

where $\hat{\Psi}_{i}=\hat{\Psi}\left(x_{i}\right), \sigma=1$ for bosons and $\sigma=-1$ for fermions. If the contraction is defined in the usual way in terms of the two-point time ordered product and normal ordered product (2.9), then the operator form of Wick's theorem holds in its usual form provided the contraction is a $c$-number [28]. In thermal field theory, one can then choose a split which satisfies

$$
\left\langle\left\langle N^{\mathrm{ES}}[\bullet]\right\rangle\right\rangle_{0}=0
$$

for all non-constant operators $\bullet$ in the interaction picture, thus mimicking the $T=0$ case (2.7). For boson fields and all contours $C$ used in thermal field theory [29], this split is given by [27] 


$$
\begin{aligned}
& \hat{\phi}^{+}=\sum_{k}\left(2 \omega_{k, \tilde{\mu}} L\right)^{-1 / 2}\left[\left(1-f_{k, \tilde{\mu}}\right) \hat{a}_{k, \tilde{\mu}} e^{-i k \cdot x}+g_{k, \tilde{\mu}} \hat{a}_{k, \tilde{\mu}}^{\dagger} e^{i k \cdot x}\right], \\
& \hat{\phi}^{-}=\sum_{k}\left(2 \omega_{k, \tilde{\mu}} L\right)^{-1 / 2}\left[f_{k, \tilde{\mu}} \hat{a}_{k, \tilde{\mu}} e^{-i k \cdot x}+\left(1-g_{k, \tilde{\mu}}\right) \hat{a}_{k, \tilde{\mu}}^{\dagger} e^{i k \cdot x}\right],
\end{aligned}
$$

where

$$
\left(1-f_{k, \tilde{\mu}}\right)\left(1-g_{k, \tilde{\mu}}\right)=1+N_{k, \tilde{\mu}}, \quad f_{k, \tilde{\mu}} g_{k, \tilde{\mu}}=-N_{k, \tilde{\mu}} .
$$

Similar expressions hold for fermion field operators [27] and also for other operators such as the momenta operator $\hat{\pi}$. Note that $N^{\mathbb{E S}}[\bullet]$, the operation we call thermal normal ordering (TNO) when (2.10) is satisfied, reduces to : $\bullet:$ at $T=0$.

We will need the free thermal boson propagator

$$
\Delta_{T}(x)=\left\langle\left\langle T_{c}[\hat{\phi}(x) \hat{\phi}(0)]\right\rangle\right\rangle_{0}=\theta_{c}(t)\left[\hat{\phi}^{+}(x), \hat{\phi}^{-}(0)\right]+\theta_{c}(-t)\left[\hat{\phi}^{+}(0), \hat{\phi}^{-}(x)\right],
$$

where $T_{c}$ means contour ordering. In the imaginary time formalism (with the contour starting at $t_{i}=0$ and ending at $\left.-i \beta\right)$, use of (2.11)-(2.13) gives

$$
\Delta_{T}(x)=-\frac{1}{4 \pi} \log \tilde{\mu}^{2} \beta^{2} Q^{2}(x)+K+\mathcal{O}(\tilde{\mu} \beta),
$$

where we have expanded in powers of $\beta \tilde{\mu} \ll 1$ and $K$ is a constant, which will not play any rôle in our analysis (see below). The $Q$ variable is given by $Q^{2}=Q_{0}^{2}+Q_{l}^{2}$ where

$$
\begin{aligned}
& Q_{0}(x, \bar{t})=-\cosh \left(\frac{\pi x}{\beta}\right) \sin \left(\frac{\pi \bar{t}}{\beta}\right), \\
& Q_{1}(x, \bar{t})=-\sinh \left(\frac{\pi x}{\beta}\right) \cos \left(\frac{\pi \bar{t}}{\beta}\right),
\end{aligned}
$$

so that $Q(x)$ is a Lorentz scalar. Observe that the boson propagator (2.14) is both ultra-violet (UV) divergent $(x \rightarrow 0)$ as well as infra-red (IR) divergent $\left(\tilde{\mu} \rightarrow 0^{+}\right)$. It also has the same form as the zero-temperature boson propagator, as for both $T \rightarrow 0^{+}$ and $x \rightarrow 0^{+}, Q_{\alpha} \rightarrow \pi T x_{\alpha}$, so that the $T=0$ propagator is recovered from (2.14) by replacing $Q_{\alpha} \rightarrow T x_{\alpha}$ For the remainder of this section we will label the propagator also by its mass $\tilde{\mu}$ so that in $(2.14), \Delta_{T}(x) \rightarrow \Delta_{T}(x ; \tilde{\mu})$.

We note here other useful properties of the $Q$ variables that will be needed later on. The first one concerns their short- and long-distance behaviour, which, from (2.16) is

$$
\begin{array}{r}
Q^{2}(x, \bar{t}) \stackrel{|x| \rightarrow \infty}{\longrightarrow} \frac{1}{4} e^{2 \pi|x| / \beta} \quad \forall \bar{t} \\
\stackrel{(x, \bar{t}) \rightarrow(0,0)}{\longrightarrow}(\pi T)^{2}\left(x^{2}+\bar{t}^{2}\right)
\end{array}
$$

and the second one is

$$
\begin{aligned}
& \epsilon^{\mu} \frac{\partial}{\partial x^{\mu}}\left[\frac{1}{\epsilon_{0}+i \epsilon_{1}} \log Z_{Q}^{+}(x)-\frac{1}{\epsilon_{0}-i \epsilon_{1}} \log Z_{Q}^{-}(x)\right]=-i \frac{\partial}{\partial x^{1}} \log Q^{2}(x), \\
& \epsilon^{\mu} \frac{\partial}{\partial x^{\mu}}\left[\frac{1}{\epsilon_{0}+i \epsilon_{1}} \log Z_{Q}^{+}(x)+\frac{1}{\epsilon_{0}-i \epsilon_{1}} \log Z_{Q}^{-}(x)\right]=\frac{\partial}{\partial x^{0}} \log Q^{2}(x)
\end{aligned}
$$


with

$$
Z_{Q}^{ \pm}(x)=Q_{0}(x) \pm i Q_{1}(x)
$$

\subsection{The sine-Gordon and Thirring models at $T>0$}

We now calculate the SG and MT partition functions through the following series of steps.

\subsubsection{Removal of $U V$ divergences in the $S G$ model by normal ordering}

Recall that for a scalar field theory in $d$ dimensions with an interaction of the form $\phi^{r}$, a diagram with $n$ vertices and $E$ external lines has a UV degree of divergence $D$ of [30],

$$
D=d-\left(\frac{d}{2}-1\right) E+n\left[\frac{r}{2}(d-2)-d\right] .
$$

Hence with $d=2, D=2-2 n$ so that other than the propagator (2.14), the only divergent diagrams $\forall r$ are tadpole diagrams. Thus, as we now show, all UV divergences are removed by thermal normal ordering the SG Hamiltonian

$$
\begin{aligned}
\hat{H}_{\mathrm{SG}} & =\int_{0}^{L} d x\left[\frac{\hat{\pi}^{2}}{2}+\frac{1}{2}\left(\frac{\partial \hat{\phi}}{\partial x}\right)^{2}-\frac{\alpha_{0}}{\lambda^{2}} \cos \lambda \hat{\phi}-\gamma_{0}\right] \\
& =: \hat{H}_{0}-\int_{0}^{L} d x\left[\frac{\alpha_{0}}{\lambda^{2}} \cos \lambda \hat{\phi}+\gamma_{0}\right] .
\end{aligned}
$$

To carry out this procedure, the Hamiltonian must be divided into a free and an interacting part. Although the term $\cos \lambda \hat{\phi}$ itself contains a mass term on expansion in powers of $\lambda$, we want to keep $\lambda$ of arbitrary size. Consider therefore

$$
\hat{H}_{\mathrm{SG}}=\left[\hat{H}_{0}+\int_{0}^{L} d x\left(\frac{1}{2} f^{2} \hat{\phi}^{2}\right)\right]-\left[\int_{0}^{L} d x\left(\frac{\alpha_{0}}{\lambda^{2}} \cos \lambda \hat{\phi}+\frac{1}{2} \rho^{2} \hat{\phi}^{2}+\gamma_{0}\right)\right],
$$

so that perturbations are about a scalar field of mass $\rho$. To take account of this fact, TNO is now denoted by $N_{\rho}^{\mathrm{ES}}[\bullet]$ and similarly we add an extra mass label to the propagators (2.14): hence we will be dealing here with $\Delta_{T}(x ; \rho)$. The link between $\rho$ and the corresponding regularisation scale in path-integral methods is discussed in Section 3.

TNO of (2.20) may be carried out by using the identity ${ }^{7}$

\footnotetext{
${ }^{7}$ These equalities may at first seem surprising, but they should be clarified by recalling that here the advanced and retarded thermal propagators are equal so that $\Delta_{T}^{>}(x-y ; \rho)=\Delta_{T}(x-y ; \rho)=\left[\hat{\phi}^{+}(x), \hat{\phi}^{-}(y)\right]$ where the positive and negative parts are given in (2.11), (2.12).
} 


$$
\begin{aligned}
e^{i \int_{c} d^{2} x j(x) \hat{\phi}(x)} & =N_{\rho}^{\mathrm{ES}}\left[e^{i \int_{c} d^{2} x j(x) \hat{\phi}(x)}\right] e^{\frac{1}{2} \int_{c} d^{2} x \int_{c} d^{2} y j(x) \Delta_{T}(x-y ; \rho) j(y)} \\
& =T_{c}\left[e^{i \int_{c} d^{2} x j(x) \hat{\phi}(x)}\right]
\end{aligned}
$$

Notice that had we used $T=0$ normal ordering, the zero-temperature propagator would have appeared in (2.21) rather than the finite temperature one: TNO means that the $Q$ variables of (2.16) are built in from the start. As in [1] we regulate the UV divergence of $\Delta_{T}(x ; \rho)$ by cutting off the theory and replace $\Delta_{T}(x ; \rho)$ by

$$
\Delta_{T}(x ; \rho ; \Lambda)=\Delta_{T}(x ; \rho)-\Delta_{T}(x ; \Lambda),
$$

where $A$ is a large mass. Note that this operation cancels the additive constant $K$ in the propagator (2.14). Now $\Delta_{T}(x ; \rho ; \Lambda)$ is both non-singular as well as $\beta$ independent for $x \rightarrow 0: \Delta_{T}(0 ; \rho ; \Lambda)=-\frac{1}{4 \pi} \ln \left(\rho^{2} / \Lambda^{2}\right)$. Also equation (2.21) with $j(x)=\lambda \delta(x-y)$ gives

$$
e^{i \lambda \hat{\phi}(y)}=\left(\frac{\rho^{2}}{\Lambda^{2}}\right)^{\lambda^{2} / 8 \pi} N_{\rho}^{\mathrm{ES}}\left[e^{i \lambda \hat{\phi}(y)}\right]
$$

so that if we define

$$
\alpha=\alpha_{0}\left(\frac{\rho^{2}}{A^{2}}\right)^{\lambda^{2} / 8 \pi}
$$

then the normal ordered form of the SG potential is just as at $T=0$ [1];

$$
\frac{\alpha_{0}}{\lambda^{2}} \cos \lambda \hat{\phi}=\frac{\alpha}{\lambda^{2}} N_{\rho}^{\mathrm{ES}}[\cos \lambda \hat{\phi}] .
$$

Also TNO of $\hat{H}_{0}$ (which is defined in (2.20) and is only equal to (2.1) for zero mass $\tilde{\mu}=0$ ) gives

$$
\hat{H}_{0}=N_{\rho}^{\mathrm{ES}}\left[\hat{H}_{0}\right]+E_{T}(\rho)
$$

where, using (2.11), (2.12),

$$
\begin{aligned}
E_{T}(\rho) & =\frac{1}{2}\left\{\left[\hat{\pi}^{+}, \hat{\pi}^{-}\right]+\left[\left(\partial_{0} \hat{\phi}\right)^{+},\left(\partial_{0} \hat{\phi}\right)^{-}\right]\right\} \\
& =\sum_{k} \frac{1}{4}\left(1+2 N_{k, \rho}\right) \frac{2 k^{2}+\rho^{2}}{\omega_{k, \rho}}=E_{0}(\rho)+\sum_{k} \frac{1}{2} N_{k, \rho}\left(\frac{2 k^{2}+\rho^{2}}{\omega_{k, \rho}}\right),
\end{aligned}
$$

and $E_{0}(\rho)$ is infinite whereas the $T$-dependent part is finite.

Thus combining (2.24) with (2.25) gives

$$
\hat{H}_{\mathrm{SG}}=N_{\rho}^{\mathrm{ES}}\left[\hat{H}_{0}-\frac{\alpha}{\lambda^{2}} \int_{0}^{L} d x \cos \lambda \hat{\phi}-L \gamma\right],
$$


where

$$
\gamma=\gamma_{0}-E_{T}(\rho)
$$

Thus $\alpha_{0}$ has been multiplicatively renormalised, $\gamma_{0}$ has been renormalised by an infinite temperature independent part appearing in $E_{T}(\rho)$ as well as having been shifted by a finite temperature dependent amount, and $\lambda$ is unchanged.

Thermal normal ordering the SG Hamiltonian has therefore absorbed all UV infinities just as zero-temperature normal ordering does [1], but it has also introduced some extra $T$-dependent finite terms. Its power will become apparent in the next section when thermal expectation values are calculated.

\subsubsection{Perturbation theory in the $S G$ model}

We now remove the IR divergences of the thermal boson propagator by introducing a mass $\tilde{\mu}$, as in (2.14), into the SG Hamiltonian (2.20). At the end of the calculation we take $\tilde{\mu} \rightarrow 0^{+}$and hence are free to add the extra mass term within the normal ordering giving the Hamiltonian

$$
\begin{aligned}
\hat{H} & =N_{\rho}^{\mathrm{ES}}\left[\hat{H}_{0}+\int_{0}^{L} d x\left(\frac{1}{2} \tilde{\mu}^{2} \hat{\phi}^{2}-\frac{\alpha}{\lambda^{2}} \cos \lambda \hat{\phi}\right)-L \gamma\right] \\
& =N_{\rho}^{\mathrm{ES}}\left[\left(\hat{H}_{0}^{\tilde{\mu}}-\gamma L\right)-\frac{\alpha}{\lambda^{2}} \int_{0}^{L} d x(\cos \lambda \hat{\phi})\right] \\
& =N_{\rho}^{\mathrm{ES}}\left[\hat{\mathcal{H}}_{0}^{\tilde{\mu}}-\frac{\alpha}{\lambda^{2}} \int_{0}^{L} d x(\cos \lambda \hat{\phi})\right] \\
& \equiv \hat{A}_{0}+\hat{A}_{I} .
\end{aligned}
$$

Here $\hat{A}_{0}$ and $\hat{A}_{I}$ denote the free $(\alpha=0)$ and interacting Hamiltonians respectively.

As usual in perturbation theory, the thermal expectation value of Heisenberg operators $\bullet_{H}$ are written in terms of interaction picture operators $\bullet$ as

$$
\langle\langle\bullet\rangle\rangle=\frac{\operatorname{Tr}\left\{e^{-\beta \hat{H}} \bullet\right\}}{\operatorname{Tr}\left\{e^{-\beta \hat{H}}\right\}}=\frac{\operatorname{Tr}\left\{e^{-\beta \hat{A}_{0}} T_{c}\left[\hat{U}\left(t_{i}-i \beta, t_{i}\right) \bullet\right]\right\}}{\operatorname{Tr}\left\{e^{-\beta \hat{A}_{0}} T_{c}\left[\hat{U}\left(t_{i}-i \beta, t_{i}\right)\right]\right\}}
$$

with times lying on the contour $C$. For $t_{i}=0$

$$
\hat{U}(-i \beta, 0)=T_{c}\left[e^{-i \int_{0}^{-i \beta} d t^{\prime \prime} \hat{A}_{l}\left(t^{\prime \prime}\right)}\right]
$$

Thus on expansion in $\hat{A}_{I}$ and working in Euclidean space, the SG partition function is given by 


$$
Z_{\mathrm{SG}}(T)=\lim _{\tilde{\mu} \rightarrow 0} \sum_{n} \frac{1}{n !} \int_{0}^{\beta} d t_{1} \ldots \int_{0}^{\beta} d t_{n} \operatorname{Tr}\left\{e^{-\beta \hat{A}_{0}} T_{c}\left[\hat{A}_{I}\left(t_{1}\right) \ldots \hat{A}_{I}\left(t_{n}\right)\right]\right\}
$$

and one is left to calculate free expectation values:

$$
\left\langle\langle\bullet\rangle_{0}^{\prime}=\frac{\operatorname{Tr}\left\{e^{-\beta \hat{A}_{0}} \bullet\right\}}{\operatorname{Tr}\left\{e^{-\beta \hat{A}_{0}}\right\}}=\frac{\operatorname{Tr}\left\{e^{-\beta N_{\rho}^{\mathrm{ES}}\left[\hat{\mathcal{H}}_{0}^{\tilde{\mu}}\right]} \bullet\right\}}{\operatorname{Tr}\left\{e^{-\beta N_{\rho}^{\mathrm{ES}}\left[\hat{\mathcal{H}}_{0}^{\tilde{\mu}}\right]}\right\}}=\frac{\operatorname{Tr}\left\{e^{-\beta N_{\rho}^{\mathrm{ES}}\left[\hat{H}_{0}^{\tilde{\mu}}\right]} \bullet\right\}}{\operatorname{Tr}\left\{e^{-\beta N_{\rho}^{\mathrm{ES}}\left[\hat{H}_{0}^{\tilde{\mu}}\right]}\right\}}\right.
$$

Here the prime label occurs because the operator appearing in the thermal weight is $N_{\rho}^{\mathrm{ES}}\left[\hat{H}_{0}^{\tilde{\mu}}\right]$, and not the simple diagonal operator : $\hat{H}_{0}^{\tilde{\mu}}: \tilde{\mu}=\sum_{k} \omega_{k, \tilde{\mu}} \hat{a}_{k, \tilde{\mu}}^{\dagger} \hat{a}_{k, \tilde{\mu}}$ as in (2.8). Since the results of [27] can only be used with this latter thermal weight, we need to manipulate $\langle\langle\rangle\rangle_{0}^{\prime}$. For that purpose, note that

$$
\hat{H}_{0}^{\tilde{\mu}}=N_{\rho}^{\mathrm{ES}}\left[\hat{H}_{0}^{\tilde{\mu}}\right]+\sum_{k} \frac{1}{4}\left(1+2 N_{k, \rho}\right)\left(\frac{2 k^{2}+\rho^{2}+\tilde{\mu}^{2}}{\omega_{k, \rho}}\right)
$$

so that from (2.3) one has

$$
N_{\rho}^{\mathrm{ES}}\left[\hat{H}_{0}^{\tilde{\mu}}\right]=: \hat{H}_{0}^{\tilde{\mu}}: \tilde{\mu}+\sum_{k} \frac{\omega_{k, \tilde{\mu}}}{2}-\sum_{k} \frac{1}{4}\left(1+2 N_{k, \rho}\right)\left(\frac{2 k^{2}+\rho^{2}+\tilde{\mu}^{2}}{\omega_{k, \rho}}\right) .
$$

Hence substituting into (2.28) gives

$$
\langle\langle\bullet\rangle\rangle_{0}^{\prime}=\frac{\operatorname{Tr}\left\{e^{-\beta: \hat{H}_{0}^{\bar{\mu}}: \dot{\mu}} \bullet\right\}}{\operatorname{Tr}\left\{e^{-\beta: \hat{H}_{0}^{\tilde{\mu}_{i}} \cdot \dot{\mu}}\right\}}=\langle\langle\bullet\rangle\rangle_{0}
$$

and the results of $[27]$ may then be used to say that $\left\langle\left\langle N_{\tilde{\mu}}^{\mathrm{ES}}[\bullet]\right\rangle\right\rangle_{0}=0$ whenever $\bullet$ is a product of operators (not including a constant). Finally, before proceeding, we note from Eq. (2.22) that

$$
N_{\rho}^{\mathrm{ES}}\left[e^{i \lambda \hat{\phi}}\right]=\left(\frac{\tilde{\mu}^{2}}{\rho^{2}}\right)^{\frac{\lambda^{2}}{8 \pi}} N_{\tilde{\mu}}^{\mathrm{ES}}\left[e^{i \lambda \hat{\phi}}\right]
$$

Following the steps in [1] for $T=0$, let us calculate at $T>0$ the free correlator

$$
\begin{aligned}
&\left\langle\left\langle T_{c} \prod_{j=1}^{n} N_{\rho}^{\mathrm{ES}}\left[e^{i \lambda_{j} \hat{\phi}\left(x_{j}\right)}\right]\right\rangle\right\rangle_{0}=\frac{\operatorname{Tr}\left\{e^{-\beta N_{\rho}^{\mathrm{ES}}\left[\tilde{H}_{0}^{\tilde{\mu}}\right]} T_{c}\left[\prod_{j=1}^{n} N_{\rho}^{\mathrm{ES}}\left[e^{i \lambda_{j} \hat{\phi}\left(x_{j}\right)}\right]\right]\right\}}{\operatorname{Tr}\left\{e^{-\beta N_{\rho}^{\mathrm{ES}}\left[\tilde{H}_{0}^{\tilde{\mu}}\right]}\right\}} \\
&=\left(\frac{\tilde{\mu}^{2}}{\rho^{2}}\right)^{\frac{\sum_{j}^{\lambda_{j}^{2}}}{8 \pi} \operatorname{Tr}\left\{e^{-\beta: H_{0}^{\tilde{\mu}}: \tilde{\mu}} T_{c}\left[\prod_{j=1}^{n} N_{\tilde{\mu}}^{\mathrm{ES}}\left[e^{i \lambda_{j} \hat{\phi}\left(x_{j}\right)}\right]\right]\right\}} \\
& \operatorname{Tr}\left\{e^{-\beta: H_{0}^{\tilde{\mu}} ; \tilde{\mu}}\right\}
\end{aligned}
$$

where we have used (2.29). Note that the above correlator is the one we would need to prove the equivalence (1.3) by expanding in $\alpha$ [1]. Also from (2.21) with $j(x)=$ 
$\lambda_{j} \delta\left(x-x_{j}\right)$ and using the fact that time ordering always puts the operators in a given order which depends only on their time arguments, one has

$$
T_{c}\left[\prod_{j=1}^{n} N_{\tilde{\mu}}^{\mathrm{ES}}\left[e^{i \lambda_{j} \hat{\phi}\left(x_{j}\right)}\right]\right]=T_{c}\left[e^{i \sum_{j}\left(\lambda_{j} \hat{\phi}\left(x_{j}\right)\right)}\right] e^{\frac{1}{2} \Delta_{T}(0 ; \tilde{\mu}) \sum_{j} \lambda_{j}^{2}}
$$

Hence

$$
\begin{aligned}
T_{c}\left[\prod_{j=1}^{n} N_{\tilde{\mu}}^{\mathrm{ES}}\left[e^{i \lambda_{j} \hat{\phi}\left(x_{j}\right)}\right]\right]= & N_{\tilde{\mu}}^{\mathrm{ES}}\left[e^{i \sum_{j}\left(\lambda_{j} \hat{\phi}\left(x_{j}\right)\right)}\right] \\
& \times e^{-\frac{1}{2} \sum_{j} \sum_{k} \lambda_{j} \Delta_{T}\left(x_{j}-x_{k} ; \tilde{\mu}\right) \lambda_{k}} e^{\frac{1}{2} \sum_{j} \lambda_{j}^{2} \Delta_{T}(0 ; \tilde{\mu})} \\
= & N_{\tilde{\mu}}^{\mathrm{ES}}\left[e^{i \sum_{j}\left(\lambda_{j} \hat{\phi}\left(x_{j}\right)\right)}\right] e^{-\sum_{j>k} \lambda_{j} \Delta_{T}\left(x_{j}-x_{k} ; \tilde{\mu}\right) \lambda_{k}} \\
= & N_{\tilde{\mu}}^{\mathrm{ES}}\left[e^{i \sum_{j}\left(\lambda_{j} \hat{\phi}\left(x_{j}\right)\right)}\right] \prod_{j=1}^{n} \prod_{j>k}\left[\beta^{2} \tilde{\mu}^{2}\left|Q\left(x_{j}-x_{k}\right)\right|^{2}\right]^{\frac{\lambda_{j} \lambda_{k}}{4 \pi}},
\end{aligned}
$$

giving, finally,

$$
\begin{aligned}
\left\langle\left\langle T_{c}\left[\prod_{j=1}^{n} N_{\rho}^{\mathrm{ES}}\left[e^{i \lambda_{j} \hat{\phi}\left(x_{j}\right)}\right]\right]\right\rangle\right\rangle_{0}= & \left(\frac{\tilde{\mu}^{2}}{\rho^{2}}\right)^{\frac{\sum_{j} \lambda_{j}^{2}}{8 \pi}}\left\langle\left\langle N_{\tilde{\mu}}^{\mathrm{ES}}\left[e^{i \sum_{j}\left(\lambda_{j} \hat{\phi}\left(x_{j}\right)\right)}\right]\right\rangle\right\rangle_{0} \\
& \times \prod_{j=1}^{n} \prod_{j>k}\left[\beta^{2} \tilde{\mu}^{2}\left|Q\left(x_{j}-x_{k}\right)\right|^{2}\right]^{\frac{\lambda_{j} \lambda_{k}}{4 \pi}} \\
= & \left(\frac{\tilde{\mu}^{2}}{\rho^{2}}\right)^{\frac{1}{8 \pi} \sum_{j} \lambda_{j}^{2}} \prod_{j=1}^{n} \prod_{j>k}\left[\beta^{2} \tilde{\mu}^{2}\left|Q\left(x_{j}-x_{k}\right)\right|^{2}\right]^{\lambda_{j} \lambda_{k} / 4 \pi},
\end{aligned}
$$

where the last line follows from expanding the exponential inside the normal ordered term and noting that all terms which contain $\hat{\phi}^{n}$ vanish (by $(2.10)$ ) apart from the one for which $n=0$, giving a contribution of 1 . It is at this stage that thermal normal ordering has been crucial for the calculation-had we used zero-temperature normal ordering, this correlator would have been very much more difficult to calculate. Note that the terms proportional to $\tilde{\mu}$ in (2.30) have a contribution

$$
\left(\tilde{\mu}^{2}\right)^{\frac{\sum_{n} \lambda_{j}^{2}}{8 \pi}} \prod_{j=1}^{n} \prod_{j>k}\left(\tilde{\mu}^{2}\right)^{\frac{\lambda_{k} \lambda_{j}}{4 \pi}}=\tilde{\mu}^{\frac{\left(\sum_{n} \lambda_{j}\right)^{2}}{4 \pi}}
$$

so unless $\sum_{n} \lambda_{j}=0$, the result vanishes as $\tilde{\mu} \rightarrow 0$, the limit in which we are interested. Hence we only consider configurations with $\sum_{n} \lambda_{j}=0$, a condition which will become analogous to the fermion chiral selection rule of Section 3. In (2.30) $n$ must therefore 
be even. If we let $m=n / 2, y_{j}=x_{j}$ for $j=n / 2+1, \ldots, n$ and define $\hat{A}_{ \pm}=N_{\rho}^{\mathrm{ES}}\left[e^{ \pm i \lambda \hat{\phi}}\right]$ then

$$
\left\langle\left\langle T_{c} \prod_{j=1}^{m} \hat{A}_{+}\left(x_{j}\right) \hat{A}_{-}\left(y_{j}\right)\right\rangle\right\rangle_{0}=\prod_{j=1}^{m} \frac{\prod_{j>k}^{m}\left[\beta^{4} \rho^{4}\left|Q\left(x_{j}-x_{k}\right)\right|^{2}\left|Q\left(y_{j}-y_{k}\right)\right|^{2}\right]^{\frac{\lambda^{2}}{4 \pi}}}{\prod_{k=1}^{m}\left[\beta^{2} \rho^{2}\left|Q\left(x_{j}-y_{k}\right)\right|^{2}\right]^{\frac{\lambda^{2}}{4 \pi}}} .
$$

To calculate the partition function we need

$$
\begin{aligned}
& \left\langle\left\langle T_{c} \prod_{j=1}^{2 n} N_{\rho}^{\mathrm{ES}}\left[\cos \lambda \hat{\phi}\left(x_{j}\right)\right]\right\rangle\right\rangle_{0} \\
& =\frac{1}{2^{2 n}} \frac{(2 n) !}{(n !)^{2}} \prod_{j=1}^{n} \frac{\prod_{j>k}^{n}\left[\beta^{4} \rho^{4}\left|Q\left(x_{j}-x_{k}\right)\right|^{2}\left|Q\left(y_{j}-y_{k}\right)\right|^{2}\right]^{\frac{\lambda^{2}}{4 \pi}}}{\prod_{k=1}^{n}\left[\beta^{2} \rho^{2}\left|Q\left(x_{j}-y_{k}\right)\right|^{2}\right]^{\frac{\lambda^{2}}{4 \pi}}},
\end{aligned}
$$

which follows from (2.32) and (2.31). Observe that the total number of $\beta \rho$ factors in this expression is $(\beta \rho)^{-\frac{\lambda^{2}}{4 \pi}(2 n)}$. Hence the partition function $(2.27)$ is

$$
\begin{aligned}
Z_{\mathrm{SG}}(T)= & \operatorname{Tr}\left\{e^{-\beta \hat{H}_{\mathrm{SG}}}\right\} \\
= & Z_{0}^{B}(T) \sum_{n} \frac{1}{(2 n) !}\left(\frac{-\alpha}{\lambda^{2}}\right)^{2 n} \\
& \times \int_{T} d^{2} x_{1} \ldots \int_{T} d^{2} x_{2 n}\left\langle\left\langle T_{c}\left[\prod_{j=1}^{2 n} N_{\rho}^{\mathrm{ES}}\left[\cos \lambda \hat{\phi}\left(t_{j}\right)\right]\right]\right\rangle_{0}\right. \\
= & Z_{0}^{B}(T) \sum_{n}\left(\frac{1}{n !}\right)^{2}\left[\frac{\alpha}{2 \lambda^{2}}\left(\frac{T}{\rho}\right)^{\frac{\lambda^{2}}{4 \pi}}\right]^{2 n} \\
& \times \prod_{j=1}^{n} \int_{T} d^{2} x_{j} \int_{T} d^{2} y_{j} \frac{\prod_{j>k}^{n}\left[\left|Q\left(x_{j}-x_{k}\right)\right|^{2}\left|Q\left(y_{j}-y_{k}\right)\right|^{2}\right]^{\frac{\lambda^{2}}{4 \pi}}}{\prod_{k=1}^{n}\left[\left|Q\left(x_{j}-y_{k}\right)\right|^{2}\right]^{\frac{\lambda^{2}}{4 \pi}}}
\end{aligned}
$$

where $Z_{0}^{B}(T)=\operatorname{Tr}\left\{e^{-\beta \hat{A}_{0}}\right\}=e^{\beta \gamma_{0} L} Z_{0}^{0, B}(T)$ and it is finite for $\gamma_{0}$ satisfying (2.26) with $\gamma$ finite.

One might wonder whether there are any extra divergences in the $x_{j}, y_{j}$ integrals in (2.33), which, for a given $n$ correspond to closed diagrams with $2 n$ vertices and an arbitrary number of loops. Recalling the asymptotic behaviour of the $Q$ variables in (2.17), one realises that the integrals in (2.33) are always finite for large spatial $x_{j}$ or $y_{j}$, because the number of $Q^{2}\left(x_{j}\right)$ powers in the denominator is always bigger than that in the numerator. As for the behaviour of the integrand when $x_{j} \rightarrow 0$ we see that the integral converges provided $\lambda^{2}<4 \pi$. Hence in this case, all the UV divergences of the theory are removed by renormalising $\alpha$. If $\lambda^{2}>4 \pi$, new divergences could 
appear in principle. However, it can be shown that as long as $\lambda^{2}<8 \pi$, those extra divergences can also be removed, by subtractions to the free energy [31]. The same argument holds in our case, since the UV divergences are $T$ independent. In fact, for $\lambda^{2}<8 \pi$ the theory is super-renormalisable [1]. For $\lambda^{2} \geqslant 8 \pi$ though, the theory is no longer super-renormalisable and indeed the ground state disappears from the spectrum [1]. These bounds should borne in mind and, although formally our expressions and results hold for any $\lambda$, they will remain finite only for $\lambda^{2}<8 \pi$ where we will assume that the $T=0$ subtractions have already been made.

\subsubsection{Perturbation theory in the massive Thirring Model}

In the Thirring model, the situation is somewhat unusual as perturbation theory not about the zero coupling $g=0$ theory, but about the massless $m_{0}=0$ theory. The correlator corresponding to (2.32) is therefore

$$
\left.\left\langle\left\langle T_{c} \prod_{j=1}^{n} \hat{\sigma}_{+}^{R}\left(x_{j}\right) \hat{\sigma}_{-}^{R}\left(y_{j}\right)\right\rangle\right\rangle\right\rangle_{\substack{m=0 \\ g \neq 0}}
$$

where $R$ stands for renormalisation and

$$
\hat{\sigma}_{ \pm}(x)=\overline{\hat{\psi}}(x) P_{ \pm} \hat{\psi}(x) \quad \text { with } \quad P_{ \pm}=\frac{1}{2}\left(1 \pm \gamma_{5}\right)
$$

At $T=0$ when the thermal expectation values are replaced by vacuum expectation values, such correlators have been calculated in a canonical operator approach by Klaiber [32]. His techniques have been used extensively by Coleman [1] as well as by others for example in the study of the Schwinger model [9]. However, the generalisation of Klaiber's work to $T>0$ does not seem to have been carried out. As in the case of the SG model, this is obtained by firstly replacing zero-temperature normal ordering by thermal normal ordering and then using thermal expectation values rather than the vacuum expectation values of [32]. This changes all zero-temperature propagators to their finite temperature analogues just as in Section 2.1.2, and is equivalent to replacing $x_{\alpha} \rightarrow \beta Q_{\alpha}$. Furthermore, the regularisation remains unchanged from the $T=0$ casethat is, given the parameter $\delta$ defined in [32],

$$
\delta=\frac{g^{2}}{4 \pi} \frac{2 \pi+g^{2}}{\pi+g^{2}},
$$

it follows from Eqs. (VII.5), (VII.4) and (VII.1) of [32] that in order for the correlator (2.34) of $\hat{\sigma}_{ \pm}(x)$ 's to be finite, these operators must be regularised as

$$
\hat{\sigma}_{+}^{R}(x)=\lim _{\varepsilon^{\prime} \rightarrow 0}\left[M \varepsilon^{\prime}\right]^{2 \delta} \hat{\psi}_{1}^{\dagger}(x) \hat{\psi}_{2}\left(x-\varepsilon^{\prime}\right) .
$$

Here the $\hat{\psi}_{i}$ 's label the components of the spinor, $\varepsilon^{\prime}$ is a 2 -vector, and $M$ in is an arbitrary renormalisation scale which, as opposed to [1], has been included so as not to alter the dimension of the operators. Finally, $\hat{\sigma}_{-}^{R}$ is given by the hermitian conjugate of (2.35). As we will verify also in Section 3.3, following these steps and using the equations of [32] mentioned above, one obtains 


$$
\begin{aligned}
& \left\langle\left\langle T_{c} \prod_{j=1}^{n} \hat{\sigma}_{+}^{R}\left(x_{j}\right) \hat{\sigma}_{-}^{R}\left(y_{j}\right)\right\rangle\right\rangle_{\substack{n=0 \\
g \neq 0}} \\
& =\left(\frac{M}{2}\right)^{2 n} \prod_{j=1}^{n} \frac{\prod_{j>k}^{n}\left[\beta^{4} M^{4}\left|Q\left(x_{j}-x_{k}\right)\right|^{2}\left|Q\left(y_{j}-y_{k}\right)\right|^{2}\right] \frac{\pi}{\pi+s^{2}}}{\prod_{k=1}^{n}\left[\beta^{2} M^{2}\left|Q\left(x_{j}-y_{k}\right)\right|^{2}\right]^{\frac{\pi}{\pi+g^{2}}}}
\end{aligned}
$$

Observe that up to the factor of $(M / 2)^{2 n}$, Eqs. (2.36) and (2.32) are identical at $T>0$ as long as the two theories are renormalised at the same scale, $M=\rho$, and provided that

$$
\frac{\pi}{\pi+g^{2}}=\frac{\lambda^{2}}{4 \pi}
$$

which is just (1.5). The partition function then follows directly, and is given by

$$
\begin{aligned}
Z_{\mathrm{MT}}(T, \mu=0)= & Z_{0}^{F}(T) \sum_{n=0}^{\infty}\left(\frac{1}{n !}\right)^{2}\left[\frac{m}{2 \beta}\left(\frac{\rho}{T}\right)^{\kappa^{2} / \pi}\right]^{2 n} \prod_{j=1}^{n} \int_{T} d^{2} x_{j} d^{2} y_{j} \\
& \times \frac{\prod_{k<j}\left[Q^{2}\left(x_{j}-x_{k}\right) Q^{2}\left(y_{j}-y_{k}\right)\right]^{1-\kappa^{2} / \pi}}{\prod_{k=1}^{n}\left[Q^{2}\left(x_{j}-y_{k}\right)\right]^{1-\kappa^{2} / \pi}} .
\end{aligned}
$$

Here $Z_{0}^{F}$ is given in $(2.5)$,

$$
\kappa^{2}=\frac{g^{2}}{1+g^{2} / \pi}
$$

and $m$ is the renormalised mass (see also Section 3 )

$$
m=m_{0}\left(\varepsilon^{\prime} \rho\right)^{-2 \varepsilon} .
$$

In perturbation theory the SG and MT model partition functions are therefore identical term by term if we identify

$$
\rho m=\frac{\alpha}{\lambda^{2}}
$$

as in (1.6).

In order to prove the relationship between arbitrary products of zero charge operators at different space-time points in the two theories and treat the $\mu \neq 0$ problem, we will turn to more powerful path-integral methods.

\section{Bosonisation with path-integral methods at $T>0$ and $\mu=0$}

Again we work in path-integral imaginary time formalism of thermal field theory and hence in Euclidean space-time with metric $(+,+)$. Now we change notation slightly so that $t=\bar{t}$ or $x^{0}$ denote the (real) Euclidean time, but, unless stated otherwise, the remaining notation follows that of [21]. As usual the exponential factor in the 
path integral is $\exp (-S)=\exp \left(-\int_{T} d^{2} x \mathcal{L}(x, t)\right)$ with $\int_{T} d^{2} x \equiv \int_{0}^{\beta} d t \int_{-\infty}^{+\infty} d x$, and the Euclidean Lagrangian densities for the $S G$ and massive Thirring models are, respectively,

$$
\begin{aligned}
\mathcal{L}_{\mathrm{SG}}[\phi] & =\frac{1}{2} \partial_{\mu} \phi \partial^{\mu} \phi-\frac{\alpha_{0}}{\lambda^{2}} \cos \lambda \phi, \\
\mathcal{L}_{\mathrm{M} \Upsilon}[\bar{\psi}, \psi] & =-\bar{\psi}\left(\not \partial+m_{0}\right) \psi+\frac{1}{2} g^{2} j_{\mu}(x) j^{\mu}(x),
\end{aligned}
$$

where we set $\gamma_{0}=0$ in the SG Lagrangian. The Euclidean gamma matrices are

$$
\gamma^{0}=\left(\begin{array}{ll}
0 & 1 \\
1 & 0
\end{array}\right), \quad \gamma^{1}=\left(\begin{array}{cc}
0 & -i \\
i & 0
\end{array}\right), \quad \gamma_{5}=-i \gamma^{0} \gamma^{1}=\left(\begin{array}{cc}
1 & 0 \\
0 & -1
\end{array}\right)
$$

so that

$$
\left\{\gamma^{\mu}, \gamma^{\nu}\right\}=2 \delta^{\mu \nu}, \quad \gamma_{\mu} \gamma_{5}=-i \epsilon_{\mu \nu} \gamma_{\nu}
$$

with $\epsilon_{01}=1$.

Let us briefly sketch the outline of this section. Firstly, in Section 3.1, some useful free boson and fermion correlators are calculated in the path-integral formalism for $T>0$ and $\mu=0$. Here we make use of the free generating functionals and thereby reproduce the results for those correlators obtained with canonical methods in the previous section. The aim of Section 3.2 is to recall how renormalisation takes place in the path integral and, in turn, to compare with our renormalisation conventions in the canonical formalism. Finally, in Section 3.3 we re-obtain the equivalence between the partition functions of the two models, now from the path-integral viewpoint. This then serves to introduce the SG and MT generating functionals which will be needed later to re-derive the correlator (2.36) in this language, as well as to introduce the auxiliary field technique we will be using extensively. Besides, most of the path-integral techniques and results needed in the rest of the paper will also be introduced in Section 3.3. As in the previous section, we always proceed by expanding around the $\alpha_{0}=m_{0}=0$ theories.

\subsection{Free generating functionals and useful correlators}

We start from the free boson Euclidean generating functional

$$
\begin{aligned}
Z_{0}^{B}[J ; T] & =N_{\beta} \int_{\text {periodic }} d \phi \exp \left\{-\left[\int_{T} d^{2} x \frac{1}{2}\left[\left(\partial_{\alpha} \phi\right)^{2}+\tilde{\mu}^{2} \phi^{2}\right]+J(x) \phi(x)\right]\right\} \\
& =Z_{0}^{B}[0 ; T] \exp \left\{\frac{1}{2} \int_{T} d^{2} x \iint_{T} d^{2} y J(x) \Delta_{T}(x-y) J(y)\right\}
\end{aligned}
$$

where the propagator is given in (2.14) for small $\tilde{\mu} \beta$ and the free boson partition function is $Z_{0}^{B}(T)=Z_{0}^{B}[0 ; T]$ as in (2.4). Note that from this section onwards the $\tilde{\mu}$ labels in propagators and partition functions will be removed since there is no possible confusion with any other mass scale. Similarly the free massless fermion generating functional is 


$$
\begin{aligned}
Z^{F}[\bar{\eta}, \eta ; T] & =N_{\beta}^{F} \int_{\text {antiper }} d \bar{\psi} d \psi \exp \left[\int_{T} d^{2} x(\bar{\psi} \not \psi \psi+\bar{\eta} \psi+\bar{\psi} \eta)\right] \\
& =Z^{F}[0,0] \exp \left[-\int_{T} d^{2} x \int_{T} d^{2} y \bar{\eta}(x) S(x-y) \eta(y)\right]
\end{aligned}
$$

where $Z^{F}[0,0]=Z_{0}^{F}$ in (2.5) and $N_{\beta}^{F}$ is the fermionic counterpart of $N_{\beta}$ in (1.7). The free massless propagator $S(x-y)$ can also be expressed in terms of the $Q$ variables as [24]

$$
S_{\alpha \beta}(x)=-\frac{1}{2 \beta} \frac{\bigotimes_{\alpha \beta}(x)}{Q^{2}(x)},
$$

where the indices $\alpha, \beta$ are Dirac indices.

The path-integral counterpart of the free correlator (2.32) is easily calculated within the path-integral formalism. We outline the main steps as they are typical of those required below. We define now $A_{ \pm}=\exp [ \pm i \lambda \phi]$ (remember that there is no operator normal ordering in the path integral), so that

$$
\left\langle\left\langle T_{c} \prod_{j=1}^{n} A_{+}\left(x_{j}\right) A_{-}\left(y_{j}\right)\right\rangle\right\rangle_{0}=\frac{Z_{0}^{B}[\tilde{J} ; T]}{Z_{0}^{B}(T)}
$$

with

$$
\tilde{J}(z)=-i \lambda \sum_{j=1}^{n}\left[\delta^{(2)}\left(z-x_{j}\right)-\delta^{(2)}\left(z-y_{j}\right)\right] .
$$

Unlike in Section 2, here the UV divergence of the propagator (2.14) will be regulated by replacing

$$
Q^{2}(0,0) \rightarrow Q^{2}\left(\varepsilon_{0}, \varepsilon_{1}\right)=T^{2} \varepsilon^{2}+\mathcal{O}\left(\varepsilon^{3}\right)
$$

where $\varepsilon_{\alpha} \rightarrow 0^{+}$and $\varepsilon^{2}=\pi^{2}\left(\varepsilon_{0}^{2}+\varepsilon_{1}^{2}\right)$. Then from (3.1), (2.14) and (3.4) it follows that

$$
\left\langle\left\langle T_{c} \prod_{j=1}^{n} A_{+}\left(x_{j}\right) A_{-}\left(y_{j}\right)\right\rangle\right\rangle_{0}=(T \varepsilon)^{n \lambda^{2} / 2 \pi} \prod_{j=1}^{n} \frac{\prod_{k<j}\left[Q^{2}\left(x_{j}-x_{k}\right) Q^{2}\left(y_{j}-y_{k}\right)\right]^{\lambda^{2} / 4 \pi}}{\prod_{k=1}^{n}\left[Q^{2}\left(x_{j}-y_{k}\right)\right]^{\lambda^{2} / 4 \pi}} .
$$

Observe that this correlator is ill defined as $\varepsilon \rightarrow 0^{+}$. Since we are still dealing with the free theory, the parameter $\lambda$ entering the definition of the $A_{ \pm}$operators above is, so far, arbitrary and, hence, the above correlator could, for instance, diverge or vanish by taking $\lambda$ purely imaginary or real respectively. This behaviour is due to the shortdistance (UV) divergent behaviour of the composite operator $\exp [i \lambda \phi(x)]$ which needs then to be renormalised (see Section 3.2 .1 below). Also note that the $\tilde{\mu}$ dependence in (3.5) has vanished for the same reasons as those of Section 2.2.2, i.e. because we 
have chosen equal numbers of $A_{+}$and $A_{-}$. It is not difficult to see that, had we chosen different numbers, the above correlator would have been proportional to $\tilde{\mu}^{\alpha}$ with $\alpha>0$ and hence it would vanish in the $\tilde{\mu} \rightarrow 0^{+}$limit. This is the bosonic counterpart of the so called fermion chiral selection rule which we will discuss below.

Similarly we need to calculate the fermion free correlator of an arbitrary number of insertions of the operators $\sigma_{ \pm}(x)=\bar{\psi}(x) P_{ \pm} \psi(x)$. To do that, recall that the massless fermion theory is invariant under chiral transformations $\psi \rightarrow \exp \left(i \alpha \gamma_{5}\right) \psi$ with $\alpha$ real arbitrary. Under such transformation $\sigma_{ \pm}(x) \rightarrow \exp ( \pm 2 i \alpha) \sigma_{ \pm}(x)$ and therefore the thermal average of a product of $\sigma_{ \pm}(x)$ operators will vanish in the massless case unless the number of $\sigma_{+}$and $\sigma_{-}$is the same. This is the chiral selection rule, which only holds for $m_{0}=0$. Following Ref. [21], the required correlator is obtained by shifting $\bar{\psi} \rightarrow \bar{\psi} \gamma^{0}$ so that, naming $\psi_{a}$ with $a=1,2$ the two components of the bispinor $\psi$, the free massless theory now decouples into two free theories for the spinors $\psi_{a}$, with propagators

$$
S_{a}(x-y)=\beta^{-1} \sum_{n \in \mathbb{Z}} \int_{-\infty}^{+\infty} \frac{d p_{1}}{2 \pi} e^{-i p \cdot(x-y)} \frac{i p_{0} \pm p}{p^{2}}=-\frac{1}{2 \beta} \frac{1}{Z_{Q}^{ \pm}(x-y)} .
$$

The,+- signs correspond to $a=1,2$ respectively and $Z_{Q}^{ \pm}$are given in (2.19). Now the transformation $\bar{\psi} \rightarrow \bar{\psi} \gamma^{0}$ takes $\sigma_{+} \rightarrow \bar{\psi}_{2} \psi_{1}$ and $\sigma_{-} \rightarrow \bar{\psi}_{1} \psi_{2}$. Recall also the thermal averaged version of Wick's theorem for fermions [21] which is

$$
\left\langle\left\langle T_{c} \prod_{j=1}^{n} \bar{\psi}_{a}\left(x_{j}\right) \psi_{a}\left(y_{j}\right)\right\rangle\right\rangle_{0}=\operatorname{det} S_{a},
$$

where the subscript 0 for fermion correlators will denote the free massless case $m_{0}=$ $g=0$ and $S_{a}$ denotes the $n \times n$ matrix with elements $\left(S_{a}\right)_{j k}=S_{a}\left(x_{j}-y_{k}\right)$. Hence, using the result $[21,24]$

$$
(-1)^{n+1} \operatorname{det} \frac{1}{Z_{Q}^{ \pm}\left(x_{j}-y_{k}\right)}=\prod_{j=1}^{n} \frac{\prod_{k<j} Z_{Q}^{ \pm}\left(x_{j}-x_{k}\right) Z_{Q}^{ \pm}\left(y_{j}-y_{k}\right)}{\prod_{k=1}^{n} Z_{Q}^{ \pm}\left(x_{j}-y_{k}\right)},
$$

where $Z_{Q}^{ \pm}$is given in (2.19) we have

$$
\left\langle\left\langle T_{c} \prod_{j=1}^{n} \sigma_{+}\left(x_{j}\right) \sigma_{-}\left(y_{j}\right)\right\rangle\right\rangle_{0}=(2 \beta)^{-2 n} \prod_{j=1}^{n} \frac{\prod_{k<j}\left[Q^{2}\left(x_{j}-x_{k}\right) Q^{2}\left(y_{j}-y_{k}\right)\right]}{\prod_{k=1}^{n}\left[Q^{2}\left(x_{j}-y_{k}\right)\right]} .
$$

Notice that the above correlator has exactly the same structure as the boson correlator (3.5). However, (3.9) is finite and well defined, unlike (3.5), since it contains no products of fields at the same point and also because there are no mixing terms between $\psi_{1}$ and $\psi_{2}$ in the Lagrangian. We will come back to this point in Section 3.2.2. 


\subsection{Renormalisation}

Here we recall the path-integral renormalisation procedure for our present case. We want to remark that our regularisation and renormalisation conventions are going to be the same as those discussed, for instance, in Ref. [21] for $T=\mu=0$. That renormalisation is independent of $T$ and $\mu$ is an expected result which will be explicitly checked throughout the rest of the paper. In addition, note that we need only to analyse renormalisation in the free $\left(\alpha_{0}=0\right) \mathrm{SG}$ model and in the massless $\left(m_{0}=0\right)$ Thirring model. The reason is that, just as with the canonical methods, we treat the SG model and the massive Thirring model always in terms of free and $m_{0}=0$ correlators, with an arbitrary number of insertions of $\left(\alpha_{0} / \lambda^{2}\right) \cos \phi(x)$ and $m_{0} \bar{\psi} \psi(x)$, respectively.

\subsubsection{Sine-Gordon model}

As commented above, the ill-defined behaviour of the free correlator (3.5) is caused by the short-distance behaviour of the free propagator in (3.4). This problem can be solved by renormalising the composite operator $\exp [i a \phi(x)]$ with $a$ arbitrary as

$$
[\exp [i a \phi(x)]]_{\mathrm{bare}}=(\varepsilon \rho)^{a^{2} / 4 \pi}[\exp [i a \phi(x)]]^{R},
$$

where $\rho$ is an arbitrary renormalisation scale. Note that this expression is analogous to (2.22) with the identification

$$
\varepsilon=\frac{1}{\Lambda}
$$

although in the operator formalism the renormalisation was carried out through TNO. Also observe that the dependence with the scale $\rho$ is the same, though it has slightly different origins - in the canonical operator approach $\rho$ corresponded to an arbitrary mass at which normal ordering was performed whereas here there is no normal ordering and $\rho$ is an arbitrary renormalisation scale.

As observed in Section 2.2.2, all the infinities that appear in the theory are just those coming from (3.10), after free-energy subtractions, provided $\lambda^{2}<8 \pi$. Those divergences can be removed by defining the renormalised coupling constant $\alpha$ as

$$
\alpha=\alpha_{0}(\varepsilon \rho)^{\lambda^{2} / 4 \pi}
$$

(compare Eq. (2.23)), and the coupling constant $\lambda$ does not need renormalisation. We will check explicitly below that with this coupling constant renormalisation all our results remain finite (for $\lambda^{2}<8 \pi$, as explained above).

\subsubsection{Thirring model}

We have seen that the $\sigma_{ \pm}(x)$ correlators are finite in the $m_{0}=g=0$ case because the two components of the bispinor do not mix in the Lagrangian. If $g \neq 0, m_{0}=0$, the chiral symmetry is still unbroken so that $\sigma_{ \pm}(x)$ correlators again appear in the same number. However, when $\bar{\psi} \rightarrow \bar{\psi} \gamma^{0},\left(\bar{\psi} \gamma^{\mu} \psi\right)^{2} \rightarrow 4 \bar{\psi}_{1} \psi_{1} \bar{\psi}_{2} \psi_{2}$ and therefore there is mixing between $\psi_{1}$ and $\psi_{2}$. Hence 


$$
\left\langle\left\langle\bar{\psi}_{2}(x) \psi_{1}(x) \bar{\psi}_{1}(y) \psi_{2}(y)\right\rangle\right\rangle \neq-\left\langle\left\langle\bar{\psi}_{2}(x) \psi_{2}(y)\right\rangle\right\rangle\left\langle\left\langle\bar{\psi}_{1}(y) \psi_{1}(x)\right\rangle\right\rangle
$$

when $g \neq 0$, so that products of fields at the same point appear and then the $\sigma_{ \pm}(x)$ correlator becomes divergent. As for the boson operator $\exp [\operatorname{ia} \phi(x)]$, the $\sigma_{ \pm}(x)$ composite operators need renormalisation. Also as in SG, those are the only infinities to consider and now they can be absorbed in the renormalised mass $m$. We anticipate here the results for such renormalisations, which we will explicitly check below,

$$
\begin{aligned}
{\left[\sigma_{ \pm}(x)\right]^{R} } & =(\varepsilon \rho)^{\kappa^{2} / \pi}\left[\sigma_{ \pm}(x)\right]_{\text {bare }} \\
m & =m_{0}(\varepsilon \rho)^{-\kappa^{2} / \pi}
\end{aligned}
$$

where both models are renormalised at the same scale $\rho$ and $\kappa$ is given in (2.38). Notice that our renormalisation conventions here for the fermion case differ from those in Section 2.2.3, which are those used in [32] (compare for instance (3.13) with (2.39)). For that reason, we have used different notations for our short-distance cutoffs, namely, $\varepsilon$ and $\varepsilon^{\prime}$. However, observe that from (3.13) and (2.39) and the definitions of $\kappa$ and $\delta$, they are related through

$$
\varepsilon^{\prime}=\varepsilon(\varepsilon \rho)^{-\frac{g^{2}}{2 \pi+g^{2}}}
$$

and since the exponent of $\varepsilon$ in the r.h.s of the above equation is positive for $\lambda^{2}<8 \pi$ according to (1.5), we can take both $\varepsilon, \varepsilon^{\prime} \rightarrow 0^{+}$consistently. In addition, we will see below that the answer for the (finite) renormalised correlators and partition functions in both canonical and path-integral approaches is the same, even though our renormalisation conventions are different, which is a necessary consistency check.

\subsection{Generating functionals and partition functions}

\subsubsection{The SG generating functional}

As usual, all correlators can be obtained from the generating functional which, in the SG model, is

$$
Z_{\mathrm{SG}}[J ; T]=N_{\beta} \int_{\text {periodic }} d \phi \exp \left\{-\left[\int_{T} d^{2} x \mathcal{L}_{\mathrm{SG}}[\phi]+J(x) \phi(x)\right]\right\},
$$

where $Z_{\mathrm{SG}}(T) \equiv Z_{\mathrm{SG}}[0 ; T]$ is the SG partition function. Expanding formally in powers of $\alpha_{0} / \lambda^{2}$ gives

$$
Z_{\mathrm{SG}}[J ; T]=\sum_{n=0}^{\infty} \frac{1}{n !}\left(\frac{\alpha_{0}}{2 \lambda^{2}}\right)^{n} \sum_{k=0}^{n}\left(\begin{array}{l}
n \\
k
\end{array}\right) \prod_{j=1}^{n} \int_{T} d^{2} x d^{2} x_{j} Z_{0}^{B}[J+\bar{J} ; T]
$$

with

$$
\bar{J}(z)=-i \lambda \sum_{j=1}^{n} s_{j} \delta^{(2)}\left(z-x_{j}\right) ; \quad s_{j}= \begin{cases}+ & j=1, \ldots, k \\ - & j=k+1, \ldots, n\end{cases}
$$


Hence, from (3.1) we have

$$
\begin{aligned}
Z_{\mathrm{SG}}[J ; T]= & Z_{0}^{B}[J ; T] \sum_{n=0}^{\infty} \frac{1}{n !}\left(\frac{\alpha_{0}}{2 \lambda^{2}}\right)^{n} \sum_{k=0}^{n}\left(\begin{array}{c}
n \\
k
\end{array}\right) \prod_{j=1}^{n} \int_{T} d^{2} x d^{2} x_{j} \exp \left\{-\frac{\lambda^{2}}{2} n \Delta_{T}(0)\right. \\
& \left.-i \lambda \sum_{j=1}^{n} s_{j} \int_{T} d^{2} x \Delta_{T}\left(x_{j}-x\right) J(x)-\lambda^{2} \sum_{j=1}^{n} \sum_{k<j} s_{j} s_{k} \Delta_{T}\left(x_{j}-x_{k}\right)\right\} .
\end{aligned}
$$

The above expression still contains both the UV $\left(\varepsilon \rightarrow 0^{+}\right)$and IR $\left(\tilde{\mu} \rightarrow 0^{+}\right)$divergences of the propagator. However, we will see that the observables and correlators of interest are finite, after renormalisation, when those limits are taken and furthermore that the $\tilde{\mu} \rightarrow 0^{+}$limit selects a particular value of $k$ in $(3.15)$, which (see below) is again equivalent to the fermion chiral selection rule.

\subsubsection{The $M T$ generating functional}

The MT model generating functional is defined, as customarily, as

$$
\begin{aligned}
& Z^{\mathrm{MT}}[\bar{\eta}, \eta ; T, \mu] \\
& \quad=N_{\beta}^{F} \int_{\text {antiper }} d \bar{\psi} d \psi \exp \left[-\int_{T} d^{2} x\left(\mathcal{L}_{\mathrm{MT}}[\bar{\psi}, \psi]+\mu j^{0}(x)-\bar{\eta} \psi-\bar{\psi} \eta\right)\right],
\end{aligned}
$$

where, for completeness, we have also included a chemical potential $\mu$ term that we will analyse in Section 5. Following Refs. [2,3,21,24], let us rewrite the quartic Thirring interaction as a "gauge-like" interaction with an auxiliary vector field $A_{\mu}$;

$$
\begin{aligned}
& Z^{\mathrm{MT}}[\bar{\eta}, \eta ; T, \mu] \\
& \quad=\frac{N_{\beta}^{F}}{Z_{A}(T)} \int_{\text {periodic }} d A_{\mu} \exp \left[-\frac{1}{2} \int_{T} d^{2} x A_{\mu}(x) A^{\mu}(x)\right] Z_{f}[A, \bar{\eta}, \eta ; T, \mu]
\end{aligned}
$$

with

$$
\begin{aligned}
& Z_{f}[A, \bar{\eta}, \eta ; T, \mu] \\
& \quad=\int_{\text {antiper }} d \bar{\psi} d \psi \exp \left[\int_{T} d^{2} x\left(\bar{\psi}\left(\not \partial+i g A(x)+m_{0}-\mu \gamma^{0}\right) \psi+\bar{\eta} \psi+\bar{\psi} \eta\right)\right]
\end{aligned}
$$

and

$$
Z_{A}(T)=\int_{\text {periodic }} d A_{\mu} \exp \left[-\frac{1}{2} \int_{T} d^{2} x A_{\mu}(x) A^{\mu}(x)\right]
$$


In the remaining of this section and in Section 4 we will concentrate only in the case $\mu=0$. The $\mu \neq 0$ case will be studied in Section 5 .

\subsubsection{The partition functions}

We now turn to reproduce the result in [24] for the partition functions of the SG and MT models, paying particular attention to UV renormalisation and to the $\tilde{\mu} \rightarrow 0^{+}$limit.

Let us start with the SG partition function, which is obtained by setting $J=0$ in (3.15). Using the explicit form of the propagator in (2.14) one finds that $Z_{\mathrm{SG}}$ is proportional to $\tilde{\mu}^{\alpha}$ with

$$
\alpha=\frac{\lambda^{2}}{4 \pi}\left(\sum_{j=1}^{n} s_{j}\right)^{2}=\frac{\lambda^{2}}{4 \pi}(2 k-n)^{2} .
$$

Hence, in the $\tilde{\mu} \rightarrow 0^{+}$limit the only term that survives in the $k$-sum is $k=n / 2$, which in turn selects only even values for $n$ in the $n$-sum. Here we also see why, in the pathintegral language, the value of $K$ in (2.14) is irrelevant: the reason is that any constant term in $\Delta_{T}$ cancels when $J=0$ in (3.15) since it is multiplied by $(2 k-n)$ in the only surviving term $k=n / 2$. Then, redefining variables as $x_{j} \rightarrow y_{j}$ for $j=n / 2+1, \ldots, n$ and $n \rightarrow 2 n$ and taking into account the definition of the renormalised coupling constant $\alpha$ in (3.11) we get

$$
\begin{aligned}
Z_{\mathrm{SG}}(T)= & Z_{0}^{B}(T) \sum_{n=0}^{\infty}\left(\frac{1}{n !}\right)^{2}\left[\frac{\alpha}{2 \lambda^{2}}\left(\frac{T}{\rho}\right)^{\lambda^{2} / 4 \pi}\right]^{2 n} \\
& \times \prod_{j=1}^{n} \int_{T} d^{2} x_{j} d^{2} y_{j} \frac{\prod_{k<j}\left[Q^{2}\left(x_{j}-x_{k}\right) Q^{2}\left(y_{j}-y_{k}\right)\right]^{\lambda^{2} / 4 \pi}}{\prod_{k=1}^{n}\left[Q^{2}\left(x_{j}-y_{k}\right)\right]^{\lambda^{2} / 4 \pi}}
\end{aligned}
$$

(compare Eq. (2.33)). As in Section 2.2.2, we again see that, at least for $\lambda^{2}<4 \pi$, all the UV divergences of the theory are removed by renormalising $\alpha$ (see our comments in Section 2.2.2 about the regime $4 \pi \leqslant \lambda^{2}<8 \pi$ ).

Our next step will be to calculate the MT model partition function and compare it with (3.19). First, we write the auxiliary vector field $A_{\mu}(x)$ as $[3,21]$

$$
A_{\mu}(x)=-\frac{1}{g}\left[\partial_{\mu} \eta(x)+i \epsilon_{\mu \nu} \partial_{\nu} \phi(x)\right]
$$

with $\eta(x)$ and $\phi(x)$ two boson fields, chosen to be periodic in Euclidean time. Then, by performing the following chiral transformation in the path-integral fermion variables:

$$
\begin{aligned}
& \psi(x)=\exp \left\{i\left[\eta(x)+\gamma_{5} \phi(x)\right]\right\} \psi^{\prime}(x), \\
& \bar{\psi}(x)=\bar{\psi}^{\prime}(x) \exp \left\{i\left[-\eta(x)+\gamma_{5} \phi(x)\right]\right\},
\end{aligned}
$$

the $\mu=0$ MT partition function, obtained by setting $\bar{\eta}=\eta=\mu=0$ in (3.17), (3.18), may be rewritten as 


$$
\begin{aligned}
Z_{\mathrm{MT}}(T)= & \frac{N_{\beta}^{F} J_{A}}{Z_{A}(T)} \int_{\text {antiper }} d \bar{\psi} d \psi \int_{\text {periodic }} d \phi d \eta \exp \left\{\int _ { T } d ^ { 2 } x \left[-\frac{1}{2 g^{2}}\left[\partial_{\mu} \eta(x)\right]^{2}\right.\right. \\
& \left.\left.+\frac{1}{2 \kappa^{2}}\left[\partial_{\mu} \phi(x)\right]^{2}+\bar{\psi}(x)\left[\not \partial+m_{0} e^{2 i \gamma_{5} \phi(x)}\right] \psi(x)\right]\right\} .
\end{aligned}
$$

Here $J_{A}$ is the Jacobian of the transformation $d A_{\mu}=J_{A} d \phi d \eta, \kappa$ is given by (2.38) and we have used that the Jacobian of the change of variable $d \bar{\psi} d \psi=J_{F} d \bar{\psi}^{\prime} d \psi^{\prime}$, which is given by the axial anomaly $[3,33,21]$

$$
J_{F}=\exp \left[\frac{1}{2 \pi} \int_{T} d^{2} x \partial_{\mu} \phi(x) \partial^{\mu} \phi(x)\right] .
$$

Finally we have relabelled the fermion variables $\psi^{\prime} \rightarrow \psi$. Note that in (3.21) the $\eta$ field is free and the only interaction term is the one proportional to $m_{0}$ which mixes the fermion fields and $\phi(x)$. Now expand in powers of $m_{0}$, note that $\bar{\psi} e^{2 i \gamma_{5} \phi} \psi=$ $\sigma_{+} e^{2 i \phi}+\sigma_{-} e^{-2 i \phi}$ and recall that, due to the fermion chiral selection rule, only terms with the same number of $\sigma_{+}$and $\sigma_{-}$contribute. The integral over $\eta$ is readily done and the boson and fermion correlators appearing in an arbitrary term in the expansion are of the form (3.5) and (3.9), though when using (3.5) in (3.21) the fields must be rescaled $\phi \rightarrow i \kappa \phi$ because of the $\left(1 / \kappa^{2}\right)$ factor in the kinetic term. Finally, recalling that $Z_{A}(T)=J_{A}\left(Z_{0}^{B}(T) / N_{\beta}\right)^{2}$ up to $T$-independent terms and then relabelling the sum and integral variables gives

$$
\begin{aligned}
Z_{\mathrm{MT}}(T)= & Z_{0}^{F}(T) \sum_{n=0}^{\infty}\left(\frac{1}{n !}\right)^{2}\left[\frac{m}{2 \beta}\left(\frac{\rho}{T}\right)^{\kappa^{2} / \pi}\right]^{2 n} \\
& \times \prod_{j=1}^{n} \int_{T} d^{2} x_{j} d^{2} y_{j} \frac{\prod_{k<j}\left[Q^{2}\left(x_{j}-x_{k}\right) Q^{2}\left(y_{j}-y_{k}\right)\right]^{1-\kappa^{2} / \pi}}{\prod_{k=1}^{n}\left[Q^{2}\left(x_{j}-y_{k}\right)\right]^{1-\kappa^{2} / \pi}},
\end{aligned}
$$

where we have renormalised $m_{0}$ as (3.13). This is just Eq. (2.37) and shows how the work in [32] simplifies in the path-integral framework. Again by comparing (3.22) with (3.19) and using (2.6) we reach the same conclusion as that of Section 2.2.3 and [24];

$$
Z_{\mathrm{MT}}(T)=Z_{\mathrm{SG}}(T)
$$

up to vacuum energy and $T$-independent terms, provided the renormalised coupling constants of both models are identified as in (1.5), (1.6).

At this point, notice that we could have expanded directly in $m_{0}$ in the original MT Lagrangian, and then every term in the expansion would have been the $g \neq 0, m_{0}=0$ correlator of a product of $\sigma_{ \pm}(x)$. Thus from (3.22) one obtains

$$
\left\langle\left\langle T_{c} \prod_{j=1}^{n} \sigma_{+}\left(x_{j}\right) \sigma_{-}\left(y_{j}\right)\right\rangle\right\rangle_{\substack{m=0 \\ g \neq 0}}
$$




$$
=\left[\frac{(T \varepsilon)^{-\kappa^{2} / \pi}}{2 \beta}\right]^{2 n} \prod_{j=1}^{n} \frac{\prod_{k<j}\left[Q^{2}\left(x_{j}-x_{k}\right) Q^{2}\left(y_{j}-y_{k}\right)\right]^{1-\kappa^{2} / \pi}}{\prod_{k=1}^{n}\left[Q^{2}\left(x_{j}-y_{k}\right)\right]^{1-\kappa^{2} / \pi}},
$$

which, as we said in Section 3.2.2, is infinite. However, with the renormalisation (3.12) for the composite operators $\sigma_{ \pm}(x)$ one obtains (2.36) with $M=\rho$ and so this is just the $T>0$ extension of the result in [32], as required (see our comments in Section 3.2 about our renormalisation conventions).

Also once again comparison of (3.24) with (3.5) gives for the renormalised correlators

$$
\left\langle\left\langle\prod_{j=1}^{n} \sigma_{+}^{R}\left(x_{j}\right) \sigma_{-}^{R}\left(y_{j}\right)\right\rangle\right\rangle_{\substack{m=0 \\ s \neq 0}}=\left(\frac{\rho}{2}\right)^{2 n}\left\langle\left\langle T_{c} \prod_{j=1}^{n} A_{+}^{R}\left(x_{j}\right) A_{-}^{R}\left(y_{j}\right)\right\rangle\right\rangle_{0}
$$

as long as (1.5) holds, which suggests the equivalence $\sigma_{ \pm}=(\rho / 2) \exp ( \pm i \lambda \phi)$. This is the $m=\alpha=0$ version of (1.3), just by combining it with (1.6). In the next section we will obtain the $m \neq 0$ version of this formula.

\section{The mass and current equivalences at $T>0$ and $\mu=0$}

In this section we show that the mass and current operator equivalences $(1.3),(1.4)$ also hold at finite temperature, i.e. for thermal averages of correlators of those operators. The condensates of $\sigma_{ \pm}$and $A_{ \pm}$are analysed in Section 4.1 whereas we consider an arbitrary chiral invariant chain of those operators in Section 4.2. The thermal current equivalence (1.4) is discussed in Section 4.3, where we make use of point-splitting regularisation.

\subsection{The mass equivalence for the condensates}

The simplest correlators to look at to prove (1.3) would be $\left\langle\left\langle A_{ \pm}(x)\right\rangle\right\rangle_{\mathrm{SG}}$ and $\left\langle\left\langle\sigma_{ \pm}(x)\right\rangle\right\rangle_{\text {MT }}$ which do not vanish for $m \neq 0$ because the chiral symmetry is broken. However, their relationship is actually a consequence of the partition functions equivalence (3.23). The reason is that they are independent of $x$ simply because of the translation invariance of the theory so that

$$
\begin{aligned}
\frac{\alpha_{0}}{\lambda^{2}}\left\langle\left\langle[\cos \lambda \phi(x)]_{\text {bare }}\right\rangle\right\rangle_{\mathrm{SG}} & =\frac{\alpha}{\lambda^{2}}\left\langle\left\langle[\cos \lambda \phi(x)]_{R}\right\rangle\right\rangle_{\mathrm{SG}}=\frac{1}{\beta L} \alpha \frac{\partial}{\partial \alpha} Z_{\mathrm{SG}}(T), \\
m_{0}\left\langle\left\langle[\bar{\psi} \psi]_{\text {bare }}\right\rangle\right\rangle_{\mathrm{MT}} & =m\left\langle\left\langle[\bar{\psi} \psi]_{R}\right\rangle\right\rangle_{\mathrm{MT}}=\frac{1}{\beta L} m \frac{\partial}{\partial m} Z_{\mathrm{MT}}(T) .
\end{aligned}
$$

In fact, the above fermion correlator is nothing but the fermion condensate which plays the rôle of the order parameter for the chiral phase transition. However, we do not expect chiral symmetry restoration at finite temperature here since Coleman's theorem prevents any continuous symmetry to be spontaneously broken in 2D [20]. 
Therefore to prove the desired equivalence for the above correlators, all we have to do is to differentiate in (3.19) and (3.22) with respect to $\alpha$ and $m$ respectively. However, it is instructive for the next sections to carry out an explicit calculation. From (1.7) and (3.14), we have

$$
\left\langle\left\langle A_{ \pm}\right\rangle\right\rangle_{\mathrm{SG}}=\frac{Z_{\mathrm{SG}}\left[J_{ \pm} ; T\right]}{Z_{\mathrm{SG}}(T)} \text { with } J_{ \pm}(x)=\mp i \lambda \delta^{(2)}(x-z) .
$$

Next use (3.15) to obtain the selection rule for this correlator: the total power of $\tilde{\mu}$ is now given by $\left(\lambda^{2} / 4 \pi\right)(1 \pm 2 k-n)^{2}$, so that only $k=(n \mp 1) / 2$ and hence odd values of $n$ in the sum, contribute now to (3.15). Then, by redefining $n \rightarrow 2 n+1$, we obtain

$$
\begin{aligned}
\left\langle\left\langle A_{ \pm}(x)\right\rangle\right\rangle_{\mathrm{SG}}= & \frac{Z_{0}^{B}(T)}{Z_{\mathrm{SG}}(T)} \sum_{n=0}^{\infty} \frac{1}{n !(n+1) !}\left[\frac{\alpha}{2 \lambda^{2}}\left(\frac{T}{\rho}\right)^{\lambda^{2} / 4 \pi}\right]^{2 n+1} \\
& \times(T \varepsilon)^{\lambda^{2} / 4 \pi} \prod_{j=1}^{n} \prod_{l=1}^{n+1} \int_{T} d^{2} x_{j} d^{2} y_{l}\left[\frac{Q^{2}\left(x_{j}-x\right)}{Q^{2}\left(y_{l}-x\right)}\right]^{\lambda^{2} / 4 \pi} \\
& \times \frac{\prod_{k<j} \prod_{k^{\prime}<l}\left[Q^{2}\left(x_{j}-x_{k}\right) Q^{2}\left(y_{l}-y_{k^{\prime}}\right)\right]^{\lambda^{2} / 4 \pi}}{\left[Q^{2}\left(x_{j}-y_{l}\right)\right]^{\lambda^{2} / 4 \pi}} .
\end{aligned}
$$

Here we have relabelled $x_{j} \longleftrightarrow y_{l}$ and used $Q^{2}(x)=Q^{2}(-x)$.

Observe that $\left\langle\left\langle A_{ \pm}(x)\right\rangle\right\rangle$ is the same for both \pm , which we could have expected by realising that both the $S G$ Lagrangian and the path-integral measure are invariant under $\phi \rightarrow-\phi$. Also the UV $\varepsilon$ dependence in (4.1) can be absorbed in the renormalisation of $\exp (i \lambda \phi)$ according to (3.10) so that once again there are no more infinities for $\lambda^{2}<4 \pi$ (see comments above). Thus we are allowed to shift the integration variables as $x_{j} \rightarrow x_{j}+x$ and $y_{j} \rightarrow y_{j}+x \forall j$, which shows explicitly that the above correlator is actually $x$ independent. The final answer is

$$
\begin{aligned}
\left\langle\left\langle A_{ \pm}^{R}(x)\right\rangle\right\rangle_{\mathrm{SG}}= & \frac{Z_{0}^{B}(T)}{Z_{\mathrm{SG}}(T)} \sum_{n=0}^{\infty} \frac{1}{n !(n+1) !}\left(\frac{\alpha}{2 \lambda^{2}}\right)^{2 n+1} \\
& \times\left(\frac{T}{\rho}\right)^{(n+1) \lambda^{2} / 2 \pi} \prod_{j=1}^{n} \prod_{l=1}^{n+1} \int_{T} d^{2} x_{j} d^{2} y_{l}\left[\frac{Q^{2}\left(x_{j}\right)}{Q^{2}\left(y_{l}\right)}\right]^{\lambda^{2} / 4 \pi} \\
& \times \frac{\prod_{k<j} \prod_{k^{\prime}<l}\left[Q^{2}\left(x_{j}-x_{k}\right) Q^{2}\left(y_{l}-y_{k^{\prime}}\right)\right]^{\lambda^{2} / 4 \pi}}{\left[Q^{2}\left(x_{j}-y_{l}\right)\right]^{\lambda^{2} / 4 \pi}},
\end{aligned}
$$

which vanishes in the free case $\alpha=0$, consistently with the discussion in Sections 2.2 .2 and 3.1. It can also be checked, order by order in the $\alpha$ expansion that the above formula indeed gives the same answer as $\left(\lambda^{2} / \beta L\right)\left(\partial Z_{\mathrm{SG}} / \partial \alpha\right)$, as explained above, which is a consistency check.

Now calculate the $\sigma_{ \pm}(x)$ correlator in the massive Thirring model. Following the same steps as those leading from (3.17) to (3.21) and taking into account that 


$$
\bar{\psi} \exp \left[2 i \gamma_{5} \phi\right] P_{ \pm} \psi=\sigma_{ \pm}(x) \exp [ \pm 2 i \phi]
$$

gives

$$
\begin{aligned}
\left\langle\left\langle\sigma_{ \pm}(x)\right\rangle\right\rangle_{\mathrm{MT}}= & \frac{Z_{0}^{F}(T)}{Z_{\mathrm{MT}}(T)} \sum_{n=0}^{\infty} \frac{m_{0}^{n}}{n !} \sum_{k=0}^{n}\left(\begin{array}{l}
n \\
k
\end{array}\right)\left[\prod_{j=1}^{k} \prod_{l=1}^{n-k} \int_{T} d^{2} x_{j} d^{2} y_{l}\right] \\
& \times\left\langle\left\langle T_{c} \sigma_{ \pm}(x) \prod_{j=1}^{k} \prod_{l=1}^{n-k} \sigma_{+}\left(x_{j}\right) \sigma_{-}\left(y_{l}\right)\right\rangle_{0}\right. \\
& \times\left\langle\left\langle T_{c} e^{\mp 2 \kappa \phi(x)} \prod_{j=1}^{k} \prod_{l=1}^{n-k} e^{-2 \kappa\left[\phi\left(x_{j}\right)-\phi\left(y_{l}\right)\right]}\right\rangle\right\rangle_{0} .
\end{aligned}
$$

The chiral selection rule then forces the choice $k=(n \mp 1) / 2$ and $n \geqslant 1$ odd so as to have the same number of $\sigma_{+}$and $\sigma_{-}$. Now let $n \rightarrow 2 n+1$, use (3.5) and (3.9) and renormalise the mass and the composite operator $\sigma_{ \pm}(x)$ as in (3.12), (3.13). One obtains

$$
\begin{aligned}
\left\langle\left\langle\sigma_{ \pm}^{R}(x)\right\rangle\right\rangle_{\mathrm{MT}}= & \frac{Z_{0}^{F}(T)}{Z_{\mathrm{MT}}(T)} \sum_{n=0}^{\infty} \frac{1}{n !(n+1) !} \frac{\rho}{2}\left(\frac{m \rho}{2}\right)^{2 n+1}(\rho \beta)^{-2\left(1-\kappa^{2} / \pi\right)(n+1)} \\
& \times \prod_{j=1}^{n} \prod_{l=1}^{n+1} \int_{T} d^{2} x_{j} d^{2} y_{l}\left[\frac{Q^{2}\left(x_{j}\right)}{Q^{2}\left(y_{l}\right)}\right]^{1-\kappa^{2} / \pi} \\
& \times \frac{\prod_{k<j} \prod_{k^{\prime}<l}\left[Q^{2}\left(x_{j}-x_{k}\right) Q^{2}\left(y_{l}-y_{k^{\prime}}\right)\right]^{1-\kappa^{2} / \pi}}{\left[Q^{2}\left(x_{j}-y_{l}\right)\right]^{1-\kappa^{2} / \pi}} .
\end{aligned}
$$

Comparison of (4.3) with (4.2) and use of the equivalences (1.5), (1.6), (2.6) and (3.23) finally gives

$$
\left\langle\left\langle\sigma_{ \pm}^{R}(x)\right\rangle\right\rangle_{\mathrm{MT}}=\frac{\rho}{2}\left\langle\left\langle A_{ \pm}^{R}(x)\right\rangle\right\rangle_{\mathrm{SG}} .
$$

As this identity depends explicitly on the scale $\rho$, it can be combined with (1.6) to get

$$
m\left\langle\left\langle[\bar{\psi}(x) \psi(x)]_{R}\right\rangle\right\rangle_{\mathrm{MT}}=\frac{\alpha}{\lambda^{2}}\left\langle\left\langle[\cos \lambda \phi(x)]_{R}\right\rangle\right\rangle_{\mathrm{SG}},
$$

which is scale independent.

\subsection{The mass equivalence for chiral invariant operators}

We now prove (1.3) for thermal correlators of an arbitrary number of $\sigma_{ \pm}$operators evaluated at different space-time points. As was commented previously, there is no need to consider the same number of $\sigma_{+}$and $\sigma_{-}$in the $m \neq 0$ case. However, such a chiral invariant combination is chosen here because then the selection rule will be just the same as for $m_{0}=0$ which clearly simplifies matters. We do not present the calculation 
for more complicated cases here since it is more involved but it goes through in the same way and leads to the expected equivalence, as we will comment later.

Once again we start from the SG model and calculate the correlator

$$
A_{N}(X ; Y) \equiv\left\langle\left\langle T_{c} \prod_{j=1}^{N} A_{+}\left(X_{j}\right) A_{-}\left(Y_{j}\right)\right\rangle\right\rangle_{\mathrm{SG}}=\frac{Z_{\mathrm{SG}}\left[J_{N} ; T\right]}{Z_{\mathrm{SG}}(T)}
$$

with

$$
J_{N}(z)=-i \lambda \sum_{j=1}^{N}\left[\delta^{(2)}\left(z-X_{j}\right)-\delta^{(2)}\left(z-Y_{j}\right)\right] .
$$

Notice that differentation of the partition function would only give global information about the above correlator, i.e. its total integral over $X_{j}$ and $Y_{j}$, which is not enough to prove the required equivalence. As before, on substitution of (4.6) into (3.15) only $k=n / 2$ survives. On the other hand, $Z_{0}^{B}\left[J_{N}, T\right]$ gives precisely the free contribution in (3.5) so that

$$
\begin{aligned}
A_{N}^{R}(X ; Y)= & \frac{Z_{0}^{B}(T)}{Z_{\mathrm{SG}}(T)} A_{N}^{R}(X ; Y)_{0} \sum_{n=0}^{\infty}\left(\frac{1}{n !}\right)^{2}\left[\frac{\alpha}{2 \lambda^{2}}\left(\frac{T}{\rho}\right)^{\lambda^{2} / 4 \pi}\right]^{2 n} \\
& \times \prod_{j=1}^{n} \int_{T} d^{2} x_{j} d^{2} y_{j} \frac{\prod_{k<j}\left[Q^{2}\left(x_{j}-x_{k}\right) Q^{2}\left(y_{j}-y_{k}\right)\right]^{\lambda^{2} / 4 \pi}}{\prod_{k=1}^{n}\left[Q^{2}\left(x_{j}-y_{k}\right)\right]^{\lambda^{2} / 4 \pi}} \\
& \times \prod_{k=1}^{N}\left[\frac{Q^{2}\left(x_{j}-X_{k}\right) Q^{2}\left(y_{j}-Y_{k}\right)}{Q^{2}\left(x_{j}-Y_{k}\right) Q^{2}\left(y_{j}-X_{k}\right)}\right]^{\lambda^{2} / 4 \pi},
\end{aligned}
$$

where, according to (3.5) and (3.10),

$$
A_{N}^{R}(X ; Y)_{0}=\left(\frac{T}{\rho}\right)^{N \lambda^{2} / 2 \pi} \prod_{j=1}^{N} \frac{\prod_{k<j}\left[Q^{2}\left(X_{j}-X_{k}\right) Q^{2}\left(Y_{j}-Y_{k}\right)\right]^{\lambda^{2} / 4 \pi}}{\prod_{k=1}^{N}\left[Q^{2}\left(X_{j}-Y_{k}\right)\right]^{\lambda^{2} / 4 \pi}}
$$

Now, in the Thirring model we have

$$
\begin{aligned}
\left\langle\left\langle T_{c}\right.\right. & \left.\left.\prod_{j=1}^{N} \sigma_{+}^{R}\left(X_{j}\right) \sigma_{-}^{R}\left(Y_{j}\right)\right\rangle\right\rangle_{\mathrm{MT}} \\
= & (\varepsilon \rho)^{2 N \kappa^{2} / \pi} \frac{Z_{0}^{F}(T)}{Z_{\mathrm{MT}}(T)} \sum_{n=0}^{\infty} \frac{m_{0}^{2 n}}{(n !)^{2}}\left[\prod_{j=1}^{n+N} \int_{T} d^{2} x_{j} d^{2} y_{j}\right] \\
& \times\left[\prod_{k=1}^{N} \delta^{(2)}\left(x_{k}-X_{k}\right) \delta^{(2)}\left(y_{k}-Y_{k}\right)\right] \\
& \times\left\langle\left\langle T_{c} \prod_{j=1}^{n+N} \sigma_{+}\left(x_{j}\right) \sigma_{-}\left(y_{j}\right)\right\rangle\right\rangle_{0}\left\langle\left\langle T_{c} \prod_{j=1}^{n+N} e^{-2 \kappa\left[\phi\left(x_{j}\right)-\phi\left(y_{j}\right)\right]}\right\rangle\right\rangle_{0}
\end{aligned}
$$




$$
\begin{aligned}
= & \frac{Z_{0}^{F}(T)}{Z_{\mathrm{MT}}(T)}\left\langle\left\langle T_{c} \prod_{j=1}^{N} \sigma_{+}^{R}\left(X_{j}\right) \sigma_{-}^{R}\left(Y_{j}\right)\right\rangle\right\rangle_{\substack{m=0 \\
g \neq 0}} \sum_{n=0}^{\infty}\left(\frac{1}{n !}\right)^{2}\left[\frac{m}{2 \beta}\left(\frac{\rho}{T}\right)^{\kappa^{2} / \pi}\right]^{2 n} \\
& \times \prod_{j=1}^{n} \int_{T} d^{2} x_{j} d^{2} y_{j} \frac{\prod_{k<j}\left[Q^{2}\left(x_{j}-x_{k}\right) Q^{2}\left(y_{j}-y_{k}\right)\right]^{1-\kappa^{2} / \pi}}{\prod_{k=1}^{n}\left[Q^{2}\left(x_{j}-y_{k}\right)\right]^{1-\kappa^{2} / \pi}} \\
& \times \prod_{k=1}^{N}\left[\frac{Q^{2}\left(x_{j}-X_{k}\right) Q^{2}\left(y_{j}-Y_{k}\right)}{Q^{2}\left(x_{j}-Y_{k}\right) Q^{2}\left(y_{j}-X_{k}\right)}\right]^{1-\kappa^{2} / \pi},
\end{aligned}
$$

where, from (3.24) and (3.12),

$$
\begin{aligned}
\left\langle\left\langle T_{c} \prod_{j=1}^{N} \sigma_{+}^{R}\left(X_{j}\right) \sigma_{-}^{R}\left(Y_{j}\right)\right\rangle\right\rangle_{\substack{m=0 \\
g \neq 0}} & \\
= & {\left[\frac{1}{2 \beta}\left(\frac{\rho}{T}\right)^{\kappa^{2} / \pi}\right]^{2 N} \prod_{j=1}^{N} \frac{\prod_{k<j}\left[Q^{2}\left(X_{j}-X_{k}\right) Q^{2}\left(Y_{j}-Y_{k}\right)\right]^{1-\kappa^{2} / \pi}}{\prod_{k=1}^{N}\left[Q^{2}\left(X_{j}-X_{k}\right)\right]^{1-\kappa^{2} / \pi}} . }
\end{aligned}
$$

Therefore, using (3.25) and the equivalences between the coupling constants and partition functions, we find the desired result

$$
\left\langle\left\langle T_{c} \prod_{j=1}^{N} \sigma_{+}^{R}\left(X_{j}\right) \sigma_{-}^{R}\left(Y_{j}\right)\right\rangle\right\rangle_{\mathrm{MT}}=\left(\frac{\rho}{2}\right)^{2 N}\left\langle\left\langle T_{c} \prod_{j=1}^{N} A_{+}^{R}\left(X_{j}\right) A_{-}^{R}\left(Y_{j}\right)\right\rangle\right\rangle_{\mathrm{SG}} .
$$

For more complicated strings of $\sigma_{ \pm}$the calculation goes through in a similar way and leads to the expected result in which $\sigma_{ \pm}^{R}\left(x_{j}\right) \rightarrow(\rho / 2) A_{ \pm}^{R}\left(x_{j}\right)$.

\subsection{The current equivalence}

We now analyse the current equivalence (1.4). When dealing with correlators including insertions of the current operator $j_{\mu}(x)$ in the Thirring model, there is an added complication since a product of fields at the same point gives a divergence. We thus have to specify how to regularise and, eventually, renormalise. To do that we follow the same approach as in $[19,25]$ for the massless case and use point-splitting regularisation. Indeed, as we will expand in the fermion mass, it will be enough to regularise the current for $m=0$. Other than that, the previous discussion about the renormalisation of the theory will remain unaltered, i.e. no further infinities will appear once we choose the proper regulator ( see below). We begin by quickly sketching how the point-splitting method is applied to this case.

\subsubsection{Point-splitting regularisation for the fermion current}

The point-splitting regularisation method [34] consists in replacing products of fermion fields

$$
\bar{\psi}_{\alpha}(x) \psi_{\beta}(x) \rightarrow \lim _{y \rightarrow x} \bar{\psi}_{\alpha}(y) \psi_{\beta}(x)
$$


where $\lim _{y \rightarrow x}^{s} f(x, y) \equiv \frac{1}{2} \lim _{y \rightarrow x}[f(x, y)+f(y, x)]$ is the symmetric limit. Clearly we can multiply any fermion bilinear by any function $F(x, y)$ such that $F(x, x)=1$. As usual, $F(x, y)$ can be determined by demanding that the different observables obey physical conditions such as current conservation or gauge invariance [34]. Consider for example the current $j_{\mu}(x)$, which is the relevant one here, and suppose we just choose $F(x, y)=1$. Recall that the relevant theory here, according to (3.18) for $\mu=0$, is that of a fermion field in the presence of an external vector field $A_{\mu}(x)$. Then classically, $j_{\mu}(x)$ is a conserved current in such a theory. However, it is easy to check that $\partial_{x}^{\mu} \lim _{y \rightarrow x}^{s} \bar{\psi}(y) \gamma_{\mu} \psi(x)=\lim _{y \rightarrow x} \frac{1}{2} A_{\mu}(x)\left[\bar{\psi}(y) \gamma_{\mu} \psi(x)-\bar{\psi}(x) \gamma_{\mu} \psi(y)\right]$, i.e. that choice for $F(x, y)$ would not lead to a classically conserved current. We see instead that the correct regularised current is

$$
j_{\mu}^{\mathrm{reg}}(x)=\lim _{y \rightarrow x} s \bar{\psi}(y) \gamma_{\mu} \psi(x) \exp \left[i g \int_{y}^{x} A_{\alpha}(\xi) d \xi^{\alpha}\right]
$$

which is classically conserved before taking the symmetric limit. Also (4.10) can be seen to satisfy the Ward identity $\partial^{\mu}\left\langle\left\langle j_{\mu}^{\text {reg }}(x)\right\rangle\right\rangle=0$, just by writing $\left\langle\left\langle\bar{\psi}_{\alpha}(x) \psi_{\beta}(y)\right\rangle\right\rangle=$ $G_{\alpha \beta}^{>}(x, y)$, the advanced exact fermion propagator, and, in addition, when dealing with a gauge theory, this definition of the regularised current is gauge invariant, another requirement the current should satisfy $[19,25,34]$.

We note that in the free case $g=0$, the point-split current yields automatically a finite result for correlators with current insertions, without any need of renormalisation. The reason is that, from (3.6), we see that the divergent part of $S_{ \pm}$always behaves like $\left(\epsilon_{0} \pm \epsilon_{1}\right)^{-1}$ with $\epsilon_{\mu}=y_{\mu}-x_{\mu}$ and hence it cancels when the symmetric limit is taken. Furthermore, we will now see that those are the only infinities also for $g \neq 0$ so that no extra renormalisations are needed and the choice (4.10) for $F(x, y)$ is the only one giving the right equivalence with the SG model.

\subsubsection{Chiral invariant mixed correlators}

The Thirring model Lagrangian and the fermion measure are invariant under charge conjugation $C$ and therefore $\left\langle\left\langle j_{\mu}(x)\right\rangle\right\rangle_{\mathrm{MT}}=0$, consistently with $\epsilon_{\mu \nu} \partial_{\nu}\langle\langle\phi(x)\rangle\rangle_{\mathrm{SG}}=0$, following just from the $\phi \rightarrow-\phi$ invariance of the SG model. Thus, the equivalence is too simple with just one insertion of the current. In this section we concentrate on the mixed propagator with one current and one $\sigma_{+} \sigma_{-}$insertion. This correlator again has the useful property that it is chiral invariant so that the selection rules are very simple. Notice that our results can then be extended without difficulty to an arbitrary string of $\sigma_{+} \sigma_{-}$and/or $j_{\mu}$ 's or even to chiral non-invariant combinations, but for clarity we restrict here to the simplest example. In turn, we will be showing that the equivalence is also true for mixed mass-current correlators.

Thus, according to the discussion in the previous section, consider the correlator

$$
F_{\mu}\left(x, z_{1}, z_{2}\right)=\left\langle\left\langle T_{c} j_{\mu}^{\mathrm{reg}}(x) \sigma_{+}\left(z_{1}\right) \sigma_{-}\left(z_{2}\right)\right\rangle\right\rangle_{\mathrm{MT}}
$$


with $j_{\mu}^{\text {reg }}(x)$ in (4.10). After similar manipulations as those of the previous sections one obtains

$$
\begin{aligned}
F_{\mu}\left(x, z_{1}, z_{2}\right)= & \frac{Z_{0}^{F}(T)}{Z_{\mathrm{MT}}(T)} \lim _{y \rightarrow x} \sum_{n=0}^{\infty} \frac{m_{0}^{2 n}}{(n !)^{2}}\left[\prod_{j=1}^{n+1} \int_{T} d^{2} x_{j} d^{2} y_{j}\right] \delta^{(2)}\left(x_{1}-z_{1}\right) \delta^{(2)}\left(y_{1}-z_{2}\right) \\
& \times \sum_{ \pm}\left\langle\left\langle T_{c} \bar{\psi}(y) \gamma_{\mu} P_{ \pm} \psi(x) \prod_{j=1}^{n+1} \sigma_{+}\left(x_{j}\right) \sigma_{-}\left(y_{j}\right)\right\rangle\right\rangle_{0} \\
& \times\left\langle\left\langle T_{c} e^{\mp \kappa[\phi(x)-\phi(y)]} e^{i \kappa \epsilon_{\alpha \beta} \int_{y}^{x} \partial^{\beta} \phi(\xi) d \xi^{\alpha}} \prod_{j=1}^{n+1} e^{-2 \kappa\left[\phi\left(x_{j}\right)-\phi\left(y_{j}\right)\right]}\right\rangle\right\rangle_{0},
\end{aligned}
$$

where the $\eta$ field dependence is the same as in (3.21) and has been integrated out. Now notice that the only pole in $(x-y)_{\mu} \equiv \epsilon_{\mu} \rightarrow 0$ is in the fermion correlator in (4.11) and that it goes like $\epsilon^{-1}$. Thus the first two exponentials in the boson free correlator in the last line can be expanded up to $\mathcal{O}(\epsilon)$. Let us analyse first the fermion free thermal correlator in (4.11). After shifting $\bar{\psi} \rightarrow \bar{\psi} \gamma^{0}$, all the required fermion correlators are of the form

$$
\begin{aligned}
& (-1)^{n+1}\left\langle\left\langle T_{c}\left[\bar{\psi}_{1}(x) \psi_{1}(y) \pm \bar{\psi}_{2}(x) \psi_{2}(y)\right] \prod_{j=1}^{n+1} \bar{\psi}_{1}\left(y_{j}\right) \psi_{1}\left(x_{j}\right) \bar{\psi}_{2}\left(x_{j}\right) \psi_{2}\left(y_{j}\right)\right\rangle\right\rangle_{0} \\
& =\left(-\frac{1}{2 \beta}\right)^{2 n+3} \frac{1}{Z_{Q}^{+}(\epsilon)} \prod_{j=1}^{n+1} \frac{\prod_{k<j}\left[Q^{2}\left(x_{j}-x_{k}\right) Q^{2}\left(y_{j}-y_{k}\right)\right]}{\prod_{k=1}^{n+1}\left[Q^{2}\left(x_{j}-y_{k}\right)\right]} \\
& \quad \times\left[1+\epsilon^{\mu} \partial_{\mu} \log \prod_{l=1}^{n+1} \frac{Z_{Q}^{+}\left(x_{l}-x\right)}{Z_{Q}^{+}\left(y_{l}-x\right)}\right] \pm\left(\begin{array}{c}
x_{j} \longleftrightarrow y_{j} \\
Z_{Q}^{+} \longleftrightarrow Z_{Q}^{-}
\end{array}\right)+\mathcal{O}\left(\epsilon^{2}\right)
\end{aligned}
$$

where $Z_{Q}^{ \pm}$are defined in (2.19), we have used (3.7) and (3.8) and we have expanded to the relevant order in $\epsilon$. As for the boson correlators, to $\mathcal{O}(\epsilon)$, we only need to calculate

$$
\begin{aligned}
& \left\langle\left\langle T_{c} \partial_{\mu}^{x} \phi(x) \prod_{j=1}^{n+1} \exp \left[-2 \kappa\left(\phi\left(x_{j}\right)-\phi\left(y_{j}\right)\right)\right]\right\rangle\right\rangle_{0} \\
& =-\left.\frac{1}{Z_{0}^{B}(T)} \partial_{\mu}^{x} \frac{\delta}{\delta J} Z_{0}[J ; T]\right|_{J=J_{\kappa}} \\
& =\frac{(\varepsilon T)^{-2(n+1) \kappa^{2} / \pi}}{2 \pi} \prod_{j=1}^{n+1} \frac{\prod_{k<j}\left[Q^{2}\left(x_{j}-x_{k}\right) Q^{2}\left(y_{j}-y_{k}\right)\right]^{-\kappa^{2} / \pi}}{\prod_{k=1}^{n+1}\left[Q^{2}\left(x_{j}-y_{k}\right)\right]^{-\kappa^{2} / \pi}}
\end{aligned}
$$




$$
\times \partial_{\mu}^{x} \log \prod_{l=1}^{n+1} \frac{Q^{2}\left(x-x_{l}\right)}{Q^{2}\left(x-y_{l}\right)}
$$

where

$$
J_{\kappa}(z)=-2 i \sum_{j=1}^{n+1}\left[\delta^{(2)}\left(z-x_{j}\right)-\delta^{(2)}\left(z-y_{j}\right)\right]
$$

Notice that by taking the partial derivative $\partial_{\mu}^{x}$ we make sure that there is no extra $\tilde{\mu}$ dependence coming from the propagator. Also recall that the UV $\varepsilon$ factor above (not to be confused with $\epsilon_{\mu}$ ) will be absorbed in the renormalisation of the mass and the $\sigma_{ \pm}$ fields as indicated in previous sections. Finally, use of (2.18) in (4.12) gives

$$
\begin{aligned}
F_{\mu}^{R}\left(x, z_{1}, z_{2}\right) & =\frac{i}{2 \pi}\left(1-\frac{\kappa^{2}}{\pi}\right) \epsilon_{\mu \nu} \frac{Z_{0}^{F}(T)}{Z_{\mathrm{MT}}(T)} \sum_{n=0}^{\infty}\left(\frac{1}{n !}\right)^{2}\left[\frac{m}{2 \beta}\left(\frac{\rho}{T}\right)^{\kappa^{2} / \pi}\right]^{2 n} \\
& \times\left\langle\left\langle T_{c} \sigma_{+}^{R}\left(z_{1}\right) \sigma_{-}^{R}\left(z_{2}\right)\right\rangle\right\rangle_{\substack{m \neq 0 \\
z_{*} \neq 0}} \prod_{j=1}^{n} \int_{T} d^{2} x_{j} d^{2} y_{j} \\
& \times \frac{\prod_{k<j}\left[Q^{2}\left(x_{j}-x_{k}\right) Q^{2}\left(y_{j}-y_{k}\right)\right]^{1-\kappa^{2} / \pi}}{\prod_{k=1}^{n}\left[Q^{2}\left(x_{j}-y_{k}\right)\right]^{1-\kappa^{2} / \pi}} \\
& \times\left[\frac{Q^{2}\left(x_{j}-z_{1}\right) Q^{2}\left(y_{j}-z_{2}\right)}{Q^{2}\left(x_{j}-z_{2}\right) Q^{2}\left(y_{j}-z_{1}\right)}\right]^{1-\frac{\kappa^{2}}{\pi}} \partial_{x}^{\nu} \log \frac{Q^{2}\left(x-z_{1}\right)}{Q^{2}\left(x-z_{2}\right)} \prod_{l=1}^{n} \frac{Q^{2}\left(x-x_{l}\right)}{Q^{2}\left(x-y_{l}\right)}
\end{aligned}
$$

with $\left\langle\left\langle T_{c} \sigma_{+}^{R}\left(z_{1}\right) \sigma_{-}^{R}\left(z_{2}\right)\right\rangle\right\rangle_{\substack{m=0 \\ g \neq 0}}$ in (4.9) for $N=1$. Notice that, thanks to (2.18), the above result is independent of the way we take the limits $\epsilon_{0} \rightarrow 0$ and $\epsilon_{1} \rightarrow 0$, which is a good check of consistency.

On the other hand, in the SG model we have to evaluate

$$
\begin{aligned}
\left\langle\left\langle T_{c} \partial_{\mu}^{x} \phi(x) A_{+}^{R}\left(z_{1}\right) A_{-}^{R}\left(z_{2}\right)\right\rangle\right\rangle_{\mathrm{SG}} \\
=-\left.(\varepsilon \rho)^{-\lambda^{2} / 2 \pi} \frac{\delta}{\delta J} Z_{\mathrm{SG}}[J ; T]\right|_{J=J_{1}} \\
=\frac{i \lambda}{4 \pi} \frac{Z_{0}^{B}(T)}{Z_{\mathrm{SG}}(T)} A_{1}^{R}\left(z_{1}, z_{2}\right)_{0} \sum_{n=0}^{\infty}\left(\frac{1}{n !}\right)^{2}\left[\frac{\alpha}{2 \lambda^{2}}\left(\frac{T}{\rho}\right)^{\lambda^{2} / 4 \pi}\right]^{2 n} \\
\quad \times \partial_{\mu}^{x} \prod_{j=1}^{n} \int_{T} d^{2} x_{j} d^{2} y_{j} \frac{\prod_{k<j}\left[Q^{2}\left(x_{j}-x_{k}\right) Q^{2}\left(y_{j}-y_{k}\right)\right]^{\lambda^{2} / 4 \pi}}{\prod_{k=1}^{n}\left[Q^{2}\left(x_{j}-y_{k}\right)\right]^{\lambda^{2} / 4 \pi}} \\
\quad \times\left[\frac{Q^{2}\left(x_{j}-z_{1}\right) Q^{2}\left(y_{j}-z_{2}\right)}{Q^{2}\left(x_{j}-z_{2}\right) Q^{2}\left(y_{j}-z_{1}\right)}\right]^{\lambda^{2} / 4 \pi} \partial_{\mu}^{x} \log \frac{Q^{2}\left(x-z_{1}\right)}{Q^{2}\left(x-z_{2}\right)} \prod_{l=1}^{n} \frac{Q^{2}\left(x-x_{l}\right)}{Q^{2}\left(x-y_{l}\right)}
\end{aligned}
$$


with $J_{1}(z)=-i \lambda\left(\delta^{(2)}\left(z-z_{1}\right)-\delta^{(2)}\left(z-z_{2}\right)\right)$ and $A_{1}^{R}\left(z_{1}, z_{2}\right)_{0}$ as in (4.7). Thus comparison of (4.14) with (4.13) and use of the equivalences we have already proved gives

$$
\left\langle\left\langle T_{c} j_{\mu}^{\mathrm{reg}}(x) \sigma_{+}^{R}\left(z_{1}\right) \sigma_{-}^{R}\left(z_{2}\right)\right\rangle\right\rangle_{\mathrm{MT}}=\left(\frac{\rho}{2}\right)^{2} \frac{\lambda}{2 \pi} \epsilon_{\mu \nu}\left\langle\left\langle T_{c} \partial_{\nu}^{x} \phi(x) A_{+}^{R}\left(z_{1}\right) A_{-}^{R}\left(z_{2}\right)\right\rangle\right\rangle_{\mathrm{SG}},
$$

which proves the current equivalence for this correlator.

\section{Bosonisation of the massive Thirring model at non-zero chemical potential}

In this section we study the thermal bosonisation of the Thirring model at non-zero chemical potential $\mu$. The chemical potential is the Lagrange multiplier associated with the conservation of the number of fermions minus anti-fermions (net fermion number) $Q_{F}=\int d x j^{0}(t, x)$. Hence, the Lagrangian density of the $\mu \neq 0$ Thirring model is just

$$
\mathcal{L}_{\mathrm{MT}}[\bar{\psi}, \psi ; \mu]=-\bar{\psi}\left(\not \partial+m_{0}\right) \psi+\frac{1}{2} g^{2} j_{\alpha}(x) j^{\alpha}(x)+\mu j^{0}(x) .
$$

By virtue of the current equivalence we have proved in Section 4.3, we can say that at $\mu=0,\left\langle\left\langle Q_{F} \bullet\right\rangle\right\rangle_{\mathrm{MT}}=\left\langle\left\langle Q_{K} \bullet\right\rangle\right\rangle_{\mathrm{SG}}$, where

$$
Q_{K}=\int_{-\infty}^{+\infty} d x \frac{\lambda}{2 \pi} \frac{\partial}{\partial x^{1}} \phi(x)=\frac{\lambda}{2 \pi}[\phi(t,+\infty)-\phi(t,-\infty)]
$$

corresponds classically to the conserved number of kinks minus anti-kinks [5]. Recall that classically $\phi_{c l}(t, \pm \infty)=2 \pi n_{ \pm} / \lambda$, with $n_{ \pm} \in \mathbb{Z}$, so that $Q_{K}\left[\phi_{c l}\right]=n_{+}-n_{-}$. We regard this equivalence as the thermal identification between Thirring fermions and SG kinks at $T \neq 0$, thus extending the $T=0$ standard result $[1,5]$, discussed in the Introduction. Physically, we interpret it as the existence of fermion excitations (the kinks) in the thermal bath.

The presence of excitations with net fermion number becomes crucial when chemical potentials are included and hence the grand-canonical ensemble partition function is considered. As the averaged net fermion number density $\rho(\mu)=(\beta L)^{-1}\left\langle\left\langle Q_{F}\right\rangle\right\rangle$ will in general be different from zero, a natural question to ask within this context is what is the bosonised version of the theory for $\mu \neq 0$. As we commented in the introduction, the answer has been given in [19] for the massless case, where the free boson partition function acquires an extra $\mu$-dependent term, unlike, for instance, the massless Schwinger model, where there are no elementary fermion excitations in the bosonised theory (all the electric charge is confined), even for non-zero $T$ and $\mu$ [19]. The Schwinger model partition function is then equivalent to that of a boson field with mass $e / \sqrt{\pi}$, with $e$ the electric charge, and the chemical potential dependence vanishes in the partition function, yielding $\rho(\mu)=0$.

Our objective in this section is then to analyse the partition function of the $\mu \neq 0$ massive Thirring model (5.1), 


$$
Z_{\mathrm{MT}}(T, \mu)=N_{\beta}^{F} \int_{\text {antiper }} d \bar{\psi} d \psi \exp \left[-\int_{T} d^{2} x \mathcal{L}_{\mathrm{MT}}[\bar{\psi}, \psi ; \mu]\right]
$$

The simplest way to proceed is to make use of the current equivalence proved in Section 4.3. Formally the argument goes as follows: we expand $Z_{\mathrm{MT}}(T, \mu)$ in $\mu$, so that every term in the expansion is a $\mu=0$ correlator of a product of $j^{0}\left(x_{j}\right)$ at different space-time points $x_{j}$. Then assume that the $T \neq 0$ current equivalence (1.4) holds for any arbitrary product of currents so that we conclude

$$
Z_{\mathrm{MT}}(T, \mu)=Z_{S G \mu}(T, \mu)
$$

with

$$
Z_{S G \mu}(T, \mu)=N_{\beta} \int_{\text {periodic }} d \phi \exp \left[-\int_{T} d^{2} x\left(\mathcal{L}_{\mathrm{SG}}-\frac{\mu \lambda}{2 \pi} \frac{\partial}{\partial x^{1}} \phi(x)\right)\right]
$$

i.e. the bosonised action is the SG model plus an extra term which is topological, in the sense that it only depends on the value of the field at the spatial boundary $(x= \pm \infty)$. As we have commented before, that term is interpreted as the result of excitations with net kink (fermion) charge being present in the thermal bath and having associated the chemical potential $\mu$ in the grand-canonical ensemble. Recall that an analogous contribution was found in the chiral Lagrangian for low-energy QCD [18] where the rôle of kinks (Thirring fermions) is played by the skyrmions (QCD baryons): the chiral Lagrangian at finite baryon density acquires, among other things, a new factor $\mu Q_{S K}$ with $Q_{S K}$ the skyrmion topological charge, analogously to (5.4). The existence of this term provides an alternative proof, based on Statistical Mechanics, of the old result that the classical kinks have their quantum counterpart in a particle with fermion number equal to one $[1,22]$ and it is indeed another example of $\mu$-dependent topological terms found in the literature [17].

The equivalence $(5.3),(5.4)$ is the central result of this section. We want to stress that, formally, we have proved it only in perturbation theory around the $m=\alpha=0$ theory. Indeed, we can perform a simple consistency check of (5.3), since for $\alpha=0$, $Z_{S G \mu}$ should give the answer for the massless Thirring model obtained in [19]. In that case, $\mathcal{L}_{\mathrm{SG}}$ in $(5.4)$ is just a free boson Lagrangian, so let

$$
\phi(x, t) \rightarrow \phi(x, t)+\frac{\mu \lambda}{2 \pi} x .
$$

As for the field measure, in the free theory the field is allowed to have $\Delta \phi \equiv \phi(L / 2, t)-$ $\phi(-L / 2, t)=c$ with $c \in \mathbb{R}$ arbitrary and $L$ the length of the system, so that the total energy still remains finite when $L \rightarrow \infty$. In other words, the free theory has trivial topology since any vacuum configuration can be reached by infinitesimal variations of the field. Therefore, we can write the path-integral measure formally as $\int_{\mathbb{R}} d c \int_{\Delta \phi=c} d \phi$. Thus, under (5.5), $\Delta \phi \rightarrow \Delta \phi+\mu \lambda L / 2 \pi$, but then, by shifting $c \rightarrow c-\mu \lambda L / 2 \pi$, the 
path-integral measure remains invariant. Therefore, by completing the square and using (1.5), we find

$$
Z_{S G \mu}^{\alpha=0}(T, \mu)=\exp \left[\beta L \frac{\mu^{2}}{2\left(\pi+g^{2}\right)}\right] Z_{0}^{B}(T),
$$

which is exactly the result found in [19] for the massless Thirring model partition function.

In what follows, we provide an alternative way of obtaining the result (5.4) without having to expand in $\mu$. For that purpose, we will make use of some of the results obtained in [19] for the massless case. Hence, let us return to the massive theory for $\mu \neq 0$ and compare the series expansion of (5.2) in $m$ with that of (5.4) in $\alpha$, in the same way as we did in previous sections for $\mu=0$. Notice that we expand now around the massless $\mu \neq 0$ theory, without expanding in $\mu$. An important remark is in order here: for every term in the $\alpha$-expansion, which is an $\alpha=0$ boson correlator, we will consider that the measure is translation invariant, as discussed before. In the mass expansion of the $\mu \neq 0$ Thirring model, we will make the same assumption whenever a boson correlator appears. Therefore, it should become clear that we are perturbing around both the $\alpha=0$ Lagrangian and path-integral measure and hence our results are valid only in perturbation theory. It remains unclear to us whether we would arrive to the same conclusion, namely (5.4), within a semi-classical approximation, i.e. perturbing now around a non-trivial classical configuration, which would be consistent for $\lambda\langle\langle 1$ $[1,22]$ but that is beyond the scope of this work and it will be analysed elsewhere.

Therefore, as in previous sections, let us introduce the auxiliary field $A_{\alpha}$ and expand in $m_{0}$ in (5.2). Then we have

$$
\begin{aligned}
Z_{\mathrm{MT}}(T, \mu)= & \frac{N_{\beta}^{F}}{Z_{A}(T)} \int_{\text {periodic }} d A_{\mu} \exp \left[-\frac{1}{2} \int_{T} d^{2} x A_{\alpha}(x) A^{\alpha}(x)\right] Z_{f}[A ; T, \mu], \\
Z_{f}[A ; T, \mu]= & \sum_{n=0}^{\infty} \frac{m_{0}^{2 n}}{(n !)^{2}} \int_{\text {antiper }} d \bar{\psi} d \psi \prod_{j=1}^{n} \int_{T} d^{2} x_{j} d^{2} y_{j} \sigma_{+}\left(x_{j}\right) \sigma_{-}\left(y_{j}\right) \\
& \times \exp \left[\int_{T} d^{2} x \bar{\psi}\left(\not+i g A(x)-\mu \gamma^{0}\right) \psi\right] .
\end{aligned}
$$

Notice that (5.7) and (5.8) are nothing but the mass expansions of (3.17) and (3.18) respectively for $\bar{\eta}=\eta=0$. In order to calculate the $m=0$ correlator in (5.8), we put back the external sources $\bar{\eta}$ and $\eta$ and take $m_{0}=0$ in (3.18) and recall the following result in [19]:

$$
Z_{f}^{m_{0}=0}[A, \bar{\eta}, \eta ; T, \mu]=\frac{Z_{0}^{F}(T, \mu)}{N_{\beta}^{F}} \exp \left[-\int_{T} d^{2} x \int_{T} d^{2} y \bar{\eta}(x) G(x, y, A ; \mu) \eta(y)\right]
$$




$$
\begin{aligned}
& \times \exp \left[-\frac{1}{2} \int_{T} d^{2} x \int_{T} d^{2} y A_{\alpha}(x) \Pi^{\alpha \beta}(x-y) A_{\beta}(y)\right] \\
& \times \exp \left[-i \frac{\mu g}{\pi} \int_{T} d^{2} x A_{0}(x)\right] .
\end{aligned}
$$

Here $Z_{0}^{F}(T, \mu)$ is the $\mu \neq 0$ free massless fermion partition function which in 2D is given by

$$
\log Z_{0}^{F}(T, \mu)=\log Z_{0}^{F}(T, 0)+\beta L \frac{\mu^{2}}{2 \pi}
$$

and

$$
\Pi_{\alpha \beta}(x-y)=\frac{g^{2}}{\pi} \beta^{-1} \sum_{n \in \mathbb{Z}} \int_{-\infty}^{+\infty} \frac{d p_{1}}{2 \pi} e^{-i p \cdot(x-y)}\left(\delta_{\alpha \beta}-\frac{p_{\alpha} p_{\beta}}{p^{2}}\right)
$$

with $p_{0}=2 \pi n T, n \in \mathbb{Z} . G(x, y, A, \mu)$ is the exact massless fermion propagator, which is given by [19]

$$
\begin{aligned}
G(x, y, A ; \mu) & =\exp \{\operatorname{ig}[\chi(x)-\chi(y)]\} S(x, y ; \mu), \\
\chi(x) & =\int_{T} d^{2} z \Delta_{T}(x-z) \phi_{z} A(z)
\end{aligned}
$$

with $A_{T}$ the free boson propagator in $(2.14)$ and $S(x, y ; \mu)$ is the free fermion $\mu \neq 0$ propagator, solution of $\left(\not-\mu \gamma^{0}\right) S(x, y ; \mu)=\delta^{(2)}(x-y)$, for which we can write

$$
S(x, y ; \mu)=\exp \left[-i\left(x^{1}-y^{1}\right) \mu \gamma^{5}\right] S(x, y ; 0) .
$$

Now redefine fields and sources as $\bar{\psi} \rightarrow \bar{\psi} \gamma^{0}$ and $\bar{\eta} \rightarrow \bar{\eta} \gamma^{0}$, so that $\bar{\eta} \psi \rightarrow \bar{\eta}_{1} \psi_{2}+\bar{\eta}_{2} \psi_{1}$ and $\bar{\psi} \eta \rightarrow \bar{\psi}_{1} \eta_{2}+\bar{\psi}_{2} \eta_{1}$ and, with the above definitions,

$$
\begin{aligned}
\bar{\eta}(x) G(x, y, A ; \mu) \eta(y) & =\sum_{a=1}^{2} \bar{\eta}_{a}(x) G_{a}(x, y, A ; \mu) \eta_{a}(y), \\
G_{ \pm}(x, y, A ; \mu) & =-\frac{1}{2 \beta} \frac{Z_{Q}^{\mp}(x-y)}{Q^{2}(x-y)} \exp \left[\chi^{ \pm}(x)-\chi^{ \pm}(y) \mp i \mu\left(x^{1}-y^{1}\right)\right],
\end{aligned}
$$

where $G_{+} \equiv G_{1}, G_{-} \equiv G_{2}, Z_{Q}^{ \pm}$are given in (2.19) and

$$
\chi^{ \pm}(x)=i g \int_{T} d^{2} z \Delta_{T}(x-z)[H(z) \pm i E(z)]
$$

with $H=\partial_{0} A_{0}+\partial_{1} A_{1}$ and $E=\partial_{0} A_{1}-\partial_{1} A_{0}$. Now, let us decompose the vector field as in (3.20), but letting now $\Delta \phi(x) \neq 0$, (see our previous discussion for the massless case), so that we have 


$$
\begin{aligned}
-\frac{1}{2} \int_{T} d^{2} x \int_{T} d^{2} y A_{\alpha}(x) I^{\alpha \beta}(x-y) A_{\beta}(y) & =\frac{1}{2 \pi} \int_{T} d^{2} x \partial_{\alpha} \phi \partial^{\alpha} \phi \\
-\frac{1}{2} \int_{T} d^{2} x A_{\alpha}(x) A^{\alpha}(x) & =-\frac{1}{2 g^{2}} \int_{T} d^{2} x\left[\partial_{\alpha} \eta \partial^{\alpha} \eta-\partial_{\alpha} \phi \partial^{\alpha} \phi\right], \\
-i \frac{\mu g}{\pi} \int_{T} d^{2} x A_{0}(x) & =-\frac{\mu}{\pi} \int_{T} d^{2} x \frac{\partial}{\partial x^{1}} \phi(x), \\
g \int_{T} d^{2} z \Delta_{T}(x-z) E(z) & =-i \phi(x)
\end{aligned}
$$

and $H$ will not play any rôle.

The final step in order to calculate the $\sigma_{+} \sigma_{-}$correlator in (5.8), is to differentiate (5.9) with respect to the sources $\bar{\eta}_{a}$ and $\eta_{a}$, after redefining both fields and sources as indicated above, that is,

$$
\begin{aligned}
\left\langle\left\langle T_{c} \prod_{j=1}^{n} \sigma_{+}\left(x_{j}\right) \sigma_{-}\left(y_{j}\right)\right\rangle\right\rangle_{A}^{m_{0}=0} \\
\quad \rightarrow\left\langle\left\langle T_{c} \bar{\psi}_{2}\left(x_{j}\right) \psi_{1}\left(x_{j}\right) \bar{\psi}_{1}\left(y_{j}\right) \psi_{2}\left(y_{j}\right)\right\rangle\right\rangle_{A}^{m_{0}=0} \\
\quad=\prod_{j=1}^{n} \frac{\delta}{\delta \eta_{1}\left(x_{j}\right)} \frac{\delta}{\delta \bar{\eta}_{2}\left(x_{j}\right)} \frac{\delta}{\delta \eta_{2}\left(y_{j}\right)} \frac{\delta}{\delta \bar{\eta}_{1}\left(y_{j}\right)} Z_{f}^{m_{0}=0}[A, \bar{\eta}, \eta ; T, \mu]
\end{aligned}
$$

where the subscript $A$ indicates the thermal expectation value in the presence of $A_{\mu}$, according to (5.8). Then, taking into account (5.10) and (5.11), we find

$$
\begin{aligned}
Z_{\mathrm{MT}}(T, \mu)= & J_{A} \frac{Z_{0}^{F}(T, \mu) Z_{0}^{B}(T)}{Z_{A}(T)} \sum_{n=0}^{\infty}\left(\frac{1}{n !}\right)^{2}\left(\frac{m_{0}}{2 \beta}\right)^{2 n} \\
& \times \int_{\text {periodic }} d \phi \exp \left[\int_{T} d^{2} x\left(\frac{1}{2 \kappa^{2}} \partial_{\alpha} \phi(x) \partial^{\alpha} \phi(x)-\frac{\mu}{\pi} \frac{\partial}{\partial x^{1}} \phi(x)\right)\right] \\
& \times \prod_{j=1}^{n} \int_{T} d^{2} x_{j} d^{2} y_{j} \frac{\prod_{k<j}\left[Q^{2}\left(x_{j}-x_{k}\right) Q^{2}\left(y_{j}-y_{k}\right)\right]}{\prod_{k=1}^{n}\left[Q^{2}\left(x_{j}-y_{k}\right)\right]} \\
& \times \exp \left\{2 i\left[\phi\left(x_{j}\right)-\phi\left(y_{j}\right)-\mu\left(x_{j}^{1}-y_{j}^{1}\right)\right]\right\}
\end{aligned}
$$

At this point, and to solve the path integral in $\phi$ above, we make use of the measure translation invariance argument discussed before and, accordingly, we shift $\phi \rightarrow i \kappa(\phi-$ $\left.i \mu \kappa x^{1} / \pi\right)$ so that the $\mu$ term is eliminated in the boson action in (5.13). Then, we can calculate the boson correlator as in previous sections and finally obtain

$$
Z_{\mathrm{MT}}(T, \mu)=Z_{\mathrm{MT}}^{m=0}(T, \mu) \sum_{n=0}^{\infty}\left(\frac{1}{n !}\right)^{2}\left[\frac{m}{2 \beta}\left(\frac{\rho}{T}\right)^{\kappa^{2} / \pi}\right]^{2 n}
$$




$$
\begin{aligned}
& \times \prod_{j=1}^{n} \int_{T} d^{2} x_{j} d^{2} y_{j} \frac{\prod_{k<j}\left[Q^{2}\left(x_{j}-x_{k}\right) Q^{2}\left(y_{j}-y_{k}\right)\right]^{1-\kappa^{2} / \pi}}{\prod_{k=1}^{n}\left[Q^{2}\left(x_{j}-y_{k}\right)\right]^{1-\kappa^{2} / \pi}} \\
& \times \exp \left[2 i \mu\left(1-\frac{\kappa^{2}}{\pi}\right)\left(x_{j}^{1}-y_{j}^{1}\right)\right]
\end{aligned}
$$

where $Z_{\mathrm{MT}}^{m=0}(T, \mu)$ is given by $(5.6)$ (recall that $Z_{0}^{B}(T)=Z_{0}^{F}(T)$ ). We readily check that the above yields (3.22) for $\mu=0$. Another consistency check is that the $\mu \neq 0$ partition function we obtain is real, which can be easily checked in (5.14) just by redefining integration variables $x_{j} \leftrightarrow y_{j}$. Besides, by taking only the lowest terms in the $m$-expansion, we have numerically checked that it is also positive definite.

Now, we expand (5.4) in $\alpha_{0}$ and perform the shift (5.5), assuming the measure invariance. Then, using (5.6) and (1.5), (1.6), we obtain exactly (5.14) and then the equivalence $(5.3),(5.4)$ follows.

\section{Conclusions and outlook}

We have studied in detail bosonisation at finite temperature and non-zero fermion chemical potential in the massive Thirring and sine-Gordon models. The main new results of this paper are the following: the extension of canonical methods to $T>0$ and $\mu=0$ to prove that the partition functions of the two models are the same; the proof, via path-integral methods of the equivalence between thermal averages of different sets of zero-charge operators in the two models; and the analysis of the $\mu \neq 0$ case where the main result is a topological new term in the bosonised SG partition function, accounting for the existence of fermion modes in the spectrum. All our results have been proved in perturbation theory around the massless Thirring model or, equivalently, the free scalar theory.

In the canonical operator proof, the use of the thermal normal ordering was crucial. Also our results for the operators (mass and current) equivalences, as well as for the $T>0, \mu \neq 0$ partition function, clearly show the existence of fermion excitations in the thermal bath. This constitutes the thermal identification between sine-Gordon kinks and Thirring fermions, so generalising the existing knowledge about this subject obtained when Statistical Mechanics features were not considered. In addition, the topological term obtained for $\mu \neq \neq 0$ is the natural analogue of a similar term found in the effective low-energy action for QCD at finite baryon density.

Among the issues which we would like to investigate further in the future is the semiclassical approach, which we have commented about in the text, especially in what concerns the $\mu \neq 0$ case and the topological action. Also, as was noted in the introduction, we would like to exploit the relationship between fermions and kinks, when these are treated as topological defects, since we believe that these $2 \mathrm{D}$ systems could become a very promising playground for testing cosmological models. The experimental realisation on Josephson junctions could then provide precise laboratory tests where, in 
particular, the results obtained in this paper could be physically probed. Other points of interest which we are looking at in more detail at the moment include a numerical analysis of the free energy and the $\mu \neq 0$ fermion density, the high temperature limit of these systems, and their behaviour near the point $\lambda^{2}=8 \pi$.

\section{Acknowledgements}

We thank Peter Landshoff both for useful arguments and for originally drawing our attention in this direction. We are grateful to Tim Evans and Ray Rivers for numerous helpful discussions and to R.F. Alvarez-Estrada for useful suggestions. A.G.N. has received support through CICYT, Spain, project AEN97-1693 and through a fellowship of MEC, Spain, and would like to thank the Imperial College Theory Group for their hospitality during the completion of this work. D.A.S. is supported by P.P.A.R.C. of the UK through a research fellowship and is a member of Girton College, Cambridge. This work was supported in part by the E.S.F.

\section{References}

[1] S. Coleman, Phys. Rev. D 11 (1975) 2088.

[2] K. Furuya, R.E. Gamboa Saravi and F.A. Schaposnik, Nucl. Phys. B 208 (1982) 159.

[3] C.M. Naón, Phys. Rev. D 31 (1985) 2035.

[4] A. Dobado, A. Gómez Nicola, A. López-Maroto and J.R. Peláez, Effective Lagrangians for the Standard Model (Springer, Berlin, 1997).

[5] S. Coleman, Aspects of Symmetry (Selected Erice Lectures) (Cambridge Univ. Press, Cambridge, 1988).

[6] R. Rajaraman, Solitons and Instantons. An Introduction to Solitons and Instantons in Quantum Field Theory (North-Holland, Amsterdam, 1982).

[7] T.H.R. Skyrme, Proc. R. Soc. London A 260 (1961) 127.

[8] E. Witten, Nucl. Phys. B 223 ( 1983) 422; B 223 (1983) 433.

[9] J. Schwinger, Phys. Rev. 128 (1962) 2425;

J. Lowenstein, A. Swieca, Ann. Phys. (N.Y.) 68 (1971) 172.

| $10 \mid$ A. Barone and G. Paternò, Physics and Applications of the Josephson Effect (Wiley, New York, 1982).

[ I I I R. Monaco, Int. J. Infrared and Millimeter Waves 11 (1990) 533.

[12] N. Marlucciello and R. Monaco, Phys. Rev. B 53 (1996) 3471; Phys. Rev. B 55 (1997) 15157.

[13] T.W.B. Kibble, J. Phys. A: Math. Gen. 9 (1976) 1387.

[14] A. Vilenkin and P. Shellard, Cosmic Strings and Other Topological Defects (Cambridge Univ. Press, Cambridge, 1994).

[15] V.M.H. Ruutu et al., Nature 382 (1996) 334.

[16] A. Gómez Nicola, R.J. Rivers and D.A. Steer, in progress, 1998.

[17] A.N. Redlich and L.C.R. Wijewardhana, Phys. Rev. Lett. 54 (1985) 970.

[18] R.F. Alvarez-Estrada and A. Gómez Nicola, Phys. Lett. B 355 (1995) 288; B 380 (1996) 491 (E).

| 19 | R.F. Alvarez-Estrada and A. Gómez Nicola, Phys. Rev. D 57 (1998) 3618.

[20] S. Coleman, Comm. Math. Phys. 31 (1973) 259

[21] J. Zinn-Justin, Quantum Field Theory and Critical Phenomena (Clarendon, Oxford, 1993).

[22] J. Goldstone and R. Jackiw, Phys. Rev. D 11 (1975) 1486.

[23] C.W. Bernard, Phys. Rev. D 9 (1974) 3312.

[24] D. Delépine, R. González Felipe and J. Weyers, Phys. Lett. B 419 (1998) 296.

\{25| F. Ruiz Ruiz and R.F. Alvarez-Estrada, Phys. Rev. D 35 (1987) 3161. 
[26] A. Gómez Nicola, R.J. Rivers and D.A. Steer, Kinks versus fermions, or the 2D sine-Gordon versus massive Thirring Models, at $T>0$ and $\mu \neq 0$, (Proc. of the 5th Int. Workshop on Thermal Field Theory, Regensburg, Germany, 10-14 August, 1998), hep-th/9809080.

[27] T.S. Evans and D.A. Steer, Nucl. Phys. B 474 (1996) 481.

[28] T.S. Evans, T.W.B. Kibble and D.A. Steer, J. Math. Phys. 39 (1998) 5726

[29] M. Le Bellac, Thermal Field Theory (Cambridge Univ. Press, Cambridge, 1996).

[30] L.H. Ryder, Quantum Field Theory, (Cambridge Univ. Press, Cambridge, 1985).

[31] D.J. Amit, Y.Y. Goldschmidt and G. Grinstein, J. Phys. A 13 (1980) 585.

132| B. Klaiber, in Lectures in Theoretical Physics, Lectures given at the Summer Institute in Theoretical Physics, University of Colorado, Boulder, CO, 1967, ed. A. Barut and W. Brittin (Gordon and Breach, New York, 1968) Vol. X, Part A.

[33] K. Fujikawa, Phys. Rev. D 29 (1984) 285.

|34| H.M. Fried, Functional Methods and Models in Quantum Field Theory (MIT Press, 1972). 